\title{
Pacific
}

Journal of

Mathematics

\section{PARAGROUPE D'ADRIAN OCNEANU ET ALGÈBRE DE KAC}

\author{
MARIE-Claude DAVid
}




\title{
PARAGROUPE D'ADRIAN OCNEANU ET ALGEBRE DE KAC
}

\author{
Marie-Claude David
}

Dans ces quelques pages, nous reprenons l'essentiel des notes manuscrites d'Adrian Ocneanu intitulées "A Galois theory for operator algebras" (1986). Nous en précisons les définitions et démontrons les théorèmes essentiels: les propriétés fondamentales du paragroupe, le résultat de classification qui est un corollaire du théorème de classification de S. Popa et la caractérisation de l'inclusion d'un facteur dans son produit croisé par une algèbre de Kac de dimension finie. Il nous a paru important que le paragroupe soit explicitement défini et que ces résultats admis par tous et souvent cités par F. Goodman, P. de la Harpe, V. Jones dans leur livre [GHJ] et par S. Popa dans ses article de classification [Popa, 1 et 2] reçoivent enfin une demonstraion exhaustive. Je me suis attachée à rédiger les démonstrations qui auraient pu être données à l'époque à deux exception près:

Le caractère d'invariant complet du paragroupe est démontré grâce aux carrés commutatifs de S. Popa.

La coassociativité du coproduit de l'algèbre de Kac (\$5) était vérifiée directement dans ma première version (Publications de l'Université ParisSud \#93-06). Claire Anantharaman a attiré mon attention sur l'article de W. Szymanski $[\mathbf{S}]$, je l'en remercie: la dualité qu'il définit me permet de donner une démonstration plus algébrique.

Parmi les développements de la théorie qui pourraient fournir d'autres démonstrations à ces résultats, on peut citer, par exemple, la théorie des bimodules d'A. Ocneanu $[\mathbf{O}]$, la théorie des secteurs $[\mathbf{L 1}, \mathbf{L 2}],[\mathbf{I 1}, \mathbf{I} 2] \ldots$

Je remercie particulièrement Vaughan Jones qui m'a encouragée à entreprendre ce travail et m'a guidée lors de nombreuses discussions. Je remercie aussi Michel Enock pour ses conseils qui m'ont aidée à achever cet article.

\section{Introduction.}

Soit $N$ un sous-facteur d'indice fini dans $M$, un facteur de type $\mathrm{II}_{1}$ dont tr est la trace finie fidèle normalisée. Soit $M_{1}$ l'algèbre oblenue par construction de base: $M_{1}$ est l'algèbre de von Neumann sur $L^{2}(M, \operatorname{tr})$ engendrée par $M$ et $e_{N}$ la projection sur $L^{2}(N, \operatorname{tr})$ [VJ1]. Si $J$ est l'involution standard de $L^{2}(M, \operatorname{tr})$, 
$M_{1}$ est égal à $J N^{\prime} J . J$ permet donc de définir un anti-automorphisme $\gamma_{0}$ de $N^{\prime} \cap M_{1}$ :

$$
\gamma_{0}(x)=J x^{*} J \quad\left(x \in N^{\prime} \cap M_{1}\right) .
$$

Plus généralement, comme M. Pimsner et S. Popa ont montré que

$$
N \subset M_{n} \subset M_{2 n+1}
$$

est isomorphe à la construction de base, on sera tenté de définir, pour $x$ élément de $N^{\prime} \cap M_{2 n+1}, \gamma_{n}(x)$ par $J_{n} x^{*} J_{n}$ où $J_{n}$ est l'involution standard de $L^{2}\left(M_{n}, \operatorname{tr}\right)$. La première partie de cet article contient les vérifications nécessaires à une définition cohérente de $\gamma_{n}$.

La deuxième partie donne la définition et les propriétés fondamentales des $\gamma_{n}$.

La troisième partie contient la démonstration de ces propriétés et une expression de $\gamma_{n}(y)$ quand $y$ est un élément de $N^{\prime} \cap M_{2 n+1}$.

La quatrième partie montre que le paragroupe (la tour dérivée munie des anti-automorphismes) est un invariant complet pour l'inclusion d'un sousfacteur de profondeur finie dans le facteur hyperfini de type $\mathrm{II}_{1}$ équivalent à l'invariant défini par S. Popa dans [Popa1], à savoir le carré commutatif canonique.

On rappelle que l'inclusion $N \subset M$ est de profondeur finie si le graphe principal est fini [GHJ, 4.1]; on obtient le graphe principal de $N \subset M$ en effaçant dans le diagramme de Bratteli de la tour dérivée ce qui s'obtient par réflexion de l'étage précédent.

La cinquième partie donne une caractérisation de l'inclusion d'un facteur de type $\mathrm{II}_{1}$ dans son produit croisé par une algèbre de Kac de dimension finie:

Soient $M$ un facteur de type $\mathrm{II}_{1}$, tr sa trace normale finie fidèle normalisée et $N$ un sous-facteur d'indice fini dans $M$. Les proposition suivantes sont équivalentes:

(a) $\quad N$ est de profondeur au plus 2 dans $M$ et $N^{\prime} \cap M$ est égal à $\mathbb{C}$.

(b) $M$ est le produit croisé de $N$ par une action extérieure d'une algèbre de $\mathrm{Kac}$ de dimension finie $\mathbb{K}$.

(c) $\quad N$ est la sous-algèbre des points fixes de $M$ sous une action extérieure d'une algèbre de Kac de dimension finie $\widehat{\mathbb{K}}$.

Une démonstration de ce résultat utilisant la méthode des secteurs se trouve dans [L2] (voir aussi [I2]). Dans [I1], on trouvera une caractérisation d'une inclusion irréductible de profondeur 2 de facteurs proprement infinis.

Un résultat semblable dans le cas où l'indice est infini est montré dans [EN]. D'autre part, si $N^{\prime} \cap M_{1}$ est commutatif, l'algèbre de Kac est un groupe fini. 


\section{Représentations des algèbres de la tour obtenue en itérant la construction de base.}

1.1. Définitions [VJ1]. Soient $M$ un facteur de type $\mathrm{II}_{1}$, tr sa trace normale finie fidèle normalisée et $N$ un sous-facteur d'indice fini dans $M$. On regarde $M$ dans sa représentation standard $\pi_{0}$ sur $L^{2}(M, \operatorname{tr})$. L'espérance conditionelle $E_{N}$ de $M$ sur $N$ définit le projecteur $e_{N}$ de $L^{2}(M, \operatorname{tr}) \operatorname{sur} L^{2}(N, \operatorname{tr})$ :

Si $\xi$ est le vecteur cyclique canonique donné par la trace, si $x$ appartient à $M$, on a:

$$
e_{N}(x \xi)=E_{N}(x) \xi
$$

La construction de base sur $N \subset M$ est la définition de l'algèbre de von Neumann $M_{1}$ sur $L^{2}(M, \operatorname{tr})$ engendrée par $M$ et $e_{N}$. On connaît donc $M_{1}$ par sa représentation fidèle $\pi_{0}$ sur $L^{2}(M, \operatorname{tr})$ qui prolonge la représentation $\pi_{0}$ de $M$ par multiplication à gauche, c'est-à-dire pour tout $a$ de $M$ et tout $x$ de $M$, on a:

$$
\pi_{0}(a)(x \xi)=(a x) \xi \text { et } \pi_{0}\left(e_{N}\right)(x \xi)=E_{N}(x) \xi .
$$

D'après [VJ1, 3.1.7], la trace canonique Tr sur $M_{1}$ est une $\left([M: N]^{-1}, M\right)$ trace, c'est-à-dire $\operatorname{Tr}$ étend tr et $\operatorname{Tr}\left(e_{N} x\right)$ est égal à $[M: N]^{-1} \operatorname{tr}(x)$ pour tout $x$ de $M$. On notera alors la trace sur $M_{1}$ comme la trace sur $M$ par tr.

1.2. La tour. D'après [VJ1, 3.1.7], on peut recommencer la construction de base à partir de l'inclusion $M \subset M_{1}$ et on obtient une algèbre de von Neumann $M_{2}$ que l'on connait par sa représentation $\pi_{1}$ sur $L^{2}\left(M_{1}, \operatorname{tr}\right)$. Les restrictions de $\pi_{1}$ à $M_{1}$ ou $M$ sont les représentation de ces algèbres qui prolongent leur action par multiplication à gauche sur $M_{1} \xi_{1}$, où $\xi_{1}$ est le vecteur cyclique canonique.

La trace sur $M_{2}$ prolonge celle de $M_{1}$ et vérifie la propriété de Markov, on la notera encore tr et ainsi pour la trace de chaque algèbre construite par construction de base. En effet, en répétant la construction de base, on obtient la tour d'algèbres:

$$
N \subset M \stackrel{e_{0}}{\complement} M_{1} \stackrel{e_{1}}{\complement} M_{2} \stackrel{e_{2}}{\complement} \cdots M_{n} \stackrel{e_{n}}{\complement} M_{n+1} \cdots
$$

On connaît $M_{n+1}$ par sa représentation $\pi_{n}$ sur $L^{2}\left(M_{n}, \operatorname{tr}\right), M_{n+1}$ est l'algèbre de von Neumann engendrée par $M_{n}$ et $e_{n}$, la projection de $L^{2}\left(M_{n}, \operatorname{tr}\right)$ sur $L^{2}\left(M_{n-1}\right.$, tr $)$.

1.3. La construction de base $N \subset M_{n} \subset M_{2 n+1}$. Dans [PiPo2], M. Pimsner et S. Popa remarquent qu'on peut définir abstraitement l'algèbre de la construction de base sur $N \subset M$ comme l'unique (à un isomorphisme 
près) facteur fini $M_{1}$, muni d'une trace $\tau$, qui contienne $M$ et une projection $e$ et vérifie

$$
\begin{aligned}
& {\left[M_{1}: M\right]=[M: N]} \\
& {[e, y]=0 \quad(y \in N)} \\
& \text { exe }=E_{N}(x) e \quad(x \in M) \\
& \tau(e x)=\left[M_{1}: M\right]^{-1} \operatorname{tr}(x) \quad(x \in M) .
\end{aligned}
$$

Ils montrent alors que l'algèbre $M_{2 n+1}$ est isomorphe à l'algèbre obtenue par la construction de base sur $N \subset M_{n}$. Il existe donc une représentation fidèle $\pi_{n}$ de $M_{2 n+1}$ sur $L^{2}\left(M_{n}\right.$, tr). Le projecteur de la construction de base est alors [PiPo2, 2.6].:

$$
f_{n}^{-1}=[M: N]^{\frac{n(n+1)}{2}}\left(e_{n} e_{n-1} \ldots e_{0}\right)\left(e_{n+1} e_{n} \ldots e_{1}\right) \ldots\left(e_{2 n} e_{2 n-1} \ldots e_{n}\right)
$$

$M_{2 n+1}$ est donc le facteur engendré par $M_{n}$ et $f_{n}^{-1}$.

Si $\xi_{n}$ est le vecteur cyclique canonique de $L^{2}\left(M_{n}, \operatorname{tr}\right)$ et $x_{n}$ un élément de $M_{n}$, on a:

$$
\pi_{n}\left(f_{n}^{-1}\right)\left(x_{n} \xi_{n}\right)=E_{N}\left(x_{n}\right) \xi_{n}
$$

et la restriction de $\pi_{n}$ à $M_{n}$ est la representation standard de $M_{n}$ sur $L^{2}\left(M_{n}, \operatorname{tr}\right)$.

1.4. D'autres représentations. On pourrait aussi regarder la construction de base sur $M_{p} \subset M_{n}, p \leq n$, qui nous donne une représentation $\pi_{n}^{p}$ de $M_{2 n-p}$ sur $L^{2}\left(M_{n}\right.$, tr); posons alors

$$
f_{n}^{p}=[M: N]^{\frac{(n-p)(n-p-1)}{2}}\left(e_{n} e_{n-1} \ldots e_{p+1}\right)\left(e_{n+1} e_{n} \ldots e_{p+2}\right) \ldots\left(e_{2 n-p-1} \ldots e_{n}\right) .
$$

$M_{2 n-p}$ est le facteur engendré par $M_{n}$ et $f_{n}^{p}$ et, pour $x_{n}$ dans $M_{n}, \pi_{n}^{p}\left(f_{n}^{p}\right)\left(x_{n} \xi_{n}\right)$ vaut $E_{M_{p}}\left(x_{n}\right) \xi_{n}$.

La restriction de $\pi_{n}$ (définie en 3) à $M_{2 n-p}$ nous donne aussi une représentation de $M_{2 n-p}$ sur $L^{2}\left(M_{n}\right.$, tr $) \ldots$ Nous allons voir dans le paragraphe suivant que $\pi_{n}^{p}$ et $\pi_{n}$ coïncident sur $M_{2 n-p}$.

\subsection{Compatibilité des représentations obtenues à partir de diffé-} rentes constructions de base. Nous commençons par fixer les notations et rappeler les règles de calcul dans les algèbres de la tour.

\subsubsection{Notations.}

a) $\alpha=[M: N]$, $a$ est la partie entière de $\alpha$ et $\alpha(n, p)=\alpha^{\frac{(n-p)(n-p-1)}{2}}$.

b) $a_{n}$ est la partie entière de $\alpha^{n}$, l'indice de $M_{n}$ dans $M_{2 n}$.

c) $g_{n}^{k}=e_{n} e_{n-1} \ldots e_{k+1} e_{k} \quad n \in \mathbb{N}, k \in \mathbb{N}, n \geq k$. 
d) $g_{n}^{k}=e_{n} e_{n+1} \ldots e_{k-1} e_{k} \quad n \in \mathbb{N}, k \in \mathbb{N}, n \leq k$

remarquons que $g_{k}^{n}=\left(g_{n}^{k}\right)^{*}$ pour tous $n$ et $k$.

e) $f_{n}^{p}=\alpha(n, p) g_{n}^{p+1} g_{n+1}^{p+2} \ldots g_{2 n-p-1}^{n} \quad n \in \mathbb{N}, p \in \mathbb{N}, n \geq p \geq-1$.

\subsubsection{Bases de Pimsner-Popa [PiPo1, 1.3].}

Il existe une famille $\left\{\lambda_{j}, 1 \leq j \leq a+1\right\}$ d'éléments de $M$, appelée base de Pimsner-Popa de $M$ sur $N$, telle que:

a) $E_{N}\left(\lambda_{j}^{*} \lambda_{k}\right)=0$ si $j \neq k$.

b) $E_{N}\left(\lambda_{j}^{*} \lambda_{j}\right)=p_{j}$ où $p_{j}$ est un projecteur de $N$ de trace $\alpha-a$ si $j=a+1$ et est l'identité sinon.

Une telle famille vérifie de plus:

c) $\lambda_{j} e_{N}$ est une isométrie partielle pour $1 \leq j \leq a+1$.

d) $\sum_{j=1}^{a+1} \lambda_{\jmath} e_{N} \lambda_{j}^{*}=1$.

e) $\sum_{j=1}^{a+1} \lambda_{j} \lambda_{j}^{*}=\alpha$.

f) Tout $y$ de $M$ admet une unique décomposition $y=\sum_{j=1}^{a+1} \lambda_{j} y_{j}$ où $y_{j}$ est un élément de $p_{j} N$ égal à $E_{N}\left(\lambda_{\jmath}^{*} y\right)$. De même $y=\sum_{j=1}^{a+1} E_{N}\left(y \lambda_{j}\right) \lambda_{\jmath}^{*}$.

g) La famille $\left\{\alpha^{1 / 2} \lambda_{i} e_{0}, 1 \leq i \leq a+1\right\}$ est une base de Pimsner-Popa de $M_{1}$ sur $M$. Plus généralement, la famille $\left\{\alpha^{(p+1) / 2} \lambda_{i} g_{0}^{p}, 1 \leq i \leq a+1\right\}$ est une base de Pimsner-Popa de $M_{p+1}$ sur $M_{p}$. (Ceci résulte de la démonstration de la proposition 1.5 de [PiPo1].)

1.5.3. Règles de calcul. On rappelle que

a) $e_{k}$ appartient à $M_{k+1}$.

b) $e_{k}$ commute avec les éléments de $M_{k-1}$.

c) $e_{k}$ commute avec $e_{h}$ si $|k-h| \geq 2$.

d) $e_{k} e_{k+1} e_{k}=\alpha^{-1} e_{k}$ et $e_{k+1} e_{k} e_{k+1}=\alpha^{-1} e_{k+1}$.

e) $e_{k+1} x e_{k+1}=E_{M_{k-1}}(x) e_{k+1}\left(x \in M_{k}\right)$.

f) $E_{M_{k}}\left(e_{k}\right)=\alpha^{-1}$.

g) $f_{n}^{p}$ appartient à $M_{2 n-p}$ et commute avec les éléments de $M_{p}$.

h) $\pi_{n}\left(f_{n}^{p}\right)\left(x_{n} \xi_{n}\right)=E_{M_{p}}\left(x_{n}\right) \xi_{n}$.

Lemme 1.5.4. $f_{n}^{p} f_{n}^{-1}=f_{n}^{-1}$.

Démonstration. Pour montrer cette égalité, nous allons faire disparaître un à un les produits du type $g_{n+k}^{p+k}$ de $f_{n}^{p}$.

Voici un procédé pour faire disparaître un projecteur:

Lemme. $g_{h}^{n+k} g_{n+k}^{k} g_{n+k+1}^{k+1}=\alpha^{-1} g_{n+k-1}^{k} g_{h}^{n+k+1} g_{n+k+1}^{k+1}$ si $h \geq n+k+1$ et 
$k \geq 0$.

Démonstration.

$$
\begin{aligned}
g_{h}^{n+k} g_{n+k}^{k} g_{n+k+1}^{k+1} & =g_{h}^{n+k+2} e_{n+k+1} e_{n+k} g_{n+k}^{k} g_{n+k+1}^{k+1} \\
& =g_{h}^{n+k+2} e_{n+k+1} e_{n+k} g_{n+k-1}^{k} e_{n+k+1} g_{n+k+1}^{k+1} \\
& =g_{h}^{n+k+2} e_{n+k+1} e_{n+k} e_{n+k+1} g_{n+k-1}^{k} g_{n+k+1}^{k+1} \\
& =\alpha^{-1} g_{h}^{n+k+2} e_{n+k+1} g_{n+k-1}^{k} g_{n+k+1}^{k+1} \\
& =\alpha^{-1} g_{n+k-1}^{k} g_{h}^{n+k+2} e_{n+k+1} g_{n+k+1}^{k+1} \\
& =\alpha^{-1} g_{n+k-1}^{k} g_{h}^{n+k+1} g_{n+k+1}^{k+1}
\end{aligned}
$$

Suite de la démonstration de 1.5.2.

Ce procédé va nous premettre de faire disparaître un à un les projecteurs de $g_{2 n-p-1}^{n}$.

$g_{2 n-p-1}^{n} f_{n}^{-1}=\alpha(n,-1)\left(g_{2 n-p-1}^{n} g_{n}^{0} g_{n+1}^{1} \ldots g_{2 n-p-2}^{n-p-2}\right) g_{2 n-p-1}^{n-p-1} \ldots g_{2 n}^{n}$

En appliquant le lemme une fois, nous obtenons d'abord:

$g_{2 n-p-1}^{n} f_{n}^{-1}=\alpha(n,-1)\left(\alpha^{-1} g_{n-1}^{0} g_{2 n-p-1}^{n+1} g_{n+1}^{1} g_{n+2}^{2} \ldots g_{2 n-p-2}^{n-p-2}\right) g_{2 n-p-1}^{n-p-1} \ldots g_{2 n}^{n}$.

En appliquant ce résultat $(n-p-2)$ fois de plus, nous faisons disparaître $g_{2 n-p-1}^{n}$.

$$
\begin{aligned}
g_{2 n-p-1}^{n} f_{n}^{-1}= & \alpha(n,-1)\left(\alpha^{-(n-p-2)} g_{n-1}^{0} g_{n}^{1} g_{n+1}^{2} \ldots\right. \\
& \left.g_{2 n-p-4}^{n-p-3} g_{2 n-p-1}^{2 n-p-2} g_{2 n-p-2}^{n-p-2}\right) g_{2 n-p-1}^{n-p-1} \ldots g_{2 n}^{n} \\
= & \alpha(n,-1)\left(\alpha^{-(n-p-1)} g_{n-1}^{0} g_{n}^{1} \ldots g_{2 n-p-3}^{n-p-3}\right) g_{2 n-p-1}^{2 n-p-1} g_{2 n-p-1}^{n-p-1} \ldots g_{2 n}^{n} \\
= & \alpha(n,-1)\left(\alpha^{-(n-p-1)} g_{n-1}^{o} g_{n}^{1} \ldots g_{2 n-p-3}^{n-p-2}\right) g_{2 n-p-1}^{n-p-1} \ldots g_{2 n}^{n} .
\end{aligned}
$$

Multiplions maintenant par $g_{2 n-p-2}^{n-1}$ :

$$
\begin{aligned}
g_{2 n-p-2}^{n-1} g_{2 n-p-1}^{n} f_{n}^{-1}=\alpha(n,-1) \alpha^{-(n-p-1)} & \\
& \left(g_{2 n-p-2}^{n-1} g_{n-1}^{0} g_{n}^{1} \ldots g_{2 n-p-3}^{n-p-2}\right) g_{2 n-p-1}^{n-p-1} \ldots g_{2 n}^{n} .
\end{aligned}
$$

Par le procédé précédent appliqué $(n-p-2)$ fois, nous avons:

$$
\begin{aligned}
g_{2 n-p-2}^{n-1} g_{2 n-p-1}^{n} f_{n}^{-1} & =\alpha(n,-1) \alpha^{-(n-p-1)} \\
& \left(\alpha^{-(n-p-2)} g_{n-2}^{0} \ldots g_{2 n-p-5}^{n-p-3} g_{2 n-p-2}^{2 n-p-3} g_{2 n-p-3}^{n-p-2}\right) g_{2 n-p-1}^{n-p-1} \ldots g_{2 n}^{n} \\
g_{2 n-p-2}^{n-1} g_{2 n-p-1}^{n} f_{n}^{-1} & =\alpha(n,-1) \alpha^{-(n-p-1)-(n-p-2)} \\
& \left(g_{n-2}^{0} g_{n-1}^{1} \ldots g_{2 n-p-5}^{n-p-3}\right) g_{2 n-p-2}^{n-p-2} g_{2 n-p-1}^{n-p-1} \ldots g_{2 n}^{n} .
\end{aligned}
$$


Après la disparition de $g_{n+1}^{p+2}$, c'est-à-dire après $(n-p-1)$ absorptions, nous obtenons

$$
f_{n}^{p} f_{n}^{-1}=\alpha(n, p) \alpha(n,-1) \alpha^{-(n-p-1)-(n-p-2) \ldots-1} g_{n}^{p+1}\left(g_{p+1}^{0}\right) g_{n+1}^{1} \ldots g_{2 n}^{n} .
$$

Comme $(n-p-1)+(n-p-2) \ldots+1=\frac{(n-p)(n-p-1)}{2}$ et $g_{n}^{p+1} g_{p+1}^{0}=g_{n}^{0}$, l'égalité est démontrée.

Nous allons vérifier maintenant que les représentations $\pi_{n}, \pi_{n}^{p}, \pi_{n}^{k}$ cö̈ncident sur $M_{2 n-p}$.

Proposition 1.5.5. Avec les notations précédentes, $\pi_{n / M_{2 n-p}}=\pi_{n}^{p}$.

Corollaire 1.5.6. Toutes représentations de la même algèbre sur le même espace obtenues à partir de différentes constructions de base sont les mêmes. Plus précisement, si $p \leq k \leq n$, alors les représentations $\pi_{n}, \pi_{n}^{p}$, $\pi_{n}^{k}$ coïncident sur $M_{2 n-p}$.

Démonstration de la proposition. On sait que $\pi_{n}^{p}$ et $\pi_{n}$ ont même restriction à $M_{n}$, c'est la représentation standard de $M_{n}$ sur $L^{2}\left(M_{n}\right.$, tr); il reste donc à comparer $\pi_{n}^{p}\left(f_{n}^{p}\right)$ et $\pi_{n}\left(f_{n}^{p}\right)$.

Si $x, y$ et $z$ sont des éléments quelconques de $M_{n}, x E_{N}(y z)$ appartient $M_{n}$ et on peut écrire:

$$
\pi_{n}^{p}\left(f_{n}^{p}\right) \pi_{n}\left(x f_{n}^{-1} y\right)\left(z \xi_{n}\right)=\pi_{n}^{p}\left(f_{n}^{p}\right)\left(x E_{N}(y z) \xi_{n}\right)=E_{M_{p}}\left(x E_{N}(y z)\right) \xi_{n} .
$$

D'où:

$$
\pi_{n}^{p}\left(f_{n}^{p}\right) \pi_{n}\left(x f_{n}^{-1} y\right)\left(z \xi_{n}\right)=E_{M_{p}}(x) E_{N}(y z) \xi_{n}=\pi_{n}\left(E_{M_{p}}(x) f_{n}^{-1} y\right)\left(z \xi_{n}\right) .
$$

Nous avons donc obtenu:

$$
\pi_{n}^{p}\left(f_{n}^{p}\right) \pi_{n}\left(x f_{n}^{-1} y\right)=\pi_{n}\left(E_{M_{p}}(x) f_{n}^{-1} y\right) \quad\left(x \in M_{n}, y \in M_{n}\right) .
$$

D'autre part, calculons $f_{n}^{p} x f_{n}^{-1} y$ :

$$
f_{n}^{p} x f_{n}^{-1} y=f_{n}^{p} x f_{n}^{p} f_{n}^{-1} y=E_{M_{p}}(x) f_{n}^{p} f_{n}^{-1} y=E_{M_{p}}(x) f_{n}^{-1} y
$$

De ces deux calculs, nous déduisons:

$$
\pi_{n}^{p}\left(f_{n}^{p}\right) \pi_{n}\left(x f_{n}^{-1} y\right)=\pi_{n}\left(f_{n}^{p} x f_{n}^{-1} y\right) \quad\left(x \in M_{n}, y \in M_{n}\right) .
$$

L'égalité $\left({ }^{*}\right)$ nous permet d'écrire:

$$
\pi_{n}^{p}\left(f_{n}^{p}\right) \pi_{n}\left(\sum_{j=1}^{a_{n}+1} \lambda_{j} f_{n}^{-1} \lambda_{j}^{*}\right)=\pi_{n}\left(f_{n}^{p} \sum_{j=1}^{a_{n}+1} \lambda_{j} f_{n}^{-1} \lambda_{j}^{*}\right) .
$$


L'égalité des éléments $\pi_{n}^{p}\left(f_{n}^{p}\right)$ et $\pi_{n}\left(f_{n}^{p}\right)$ découle alors de la propriété de la base de Pimsner-Popa (1.5.2d) qui donne l'expression de l'identité de $M_{n}$ :

$$
\sum_{j-1}^{a_{n}+1} \lambda_{j} f_{n}^{-1} \lambda_{j}^{*}=1 .
$$

La compatibilité des différentes représentations nous permet de définir sans ambiguïté les anti-automorphismes associés à la tour derivée.

\section{Anti-automorphismes associés à la tour dérivée.}

2.1. Définitions et notations. On reprend ici [VJ1,3] et on rappelle que $J_{n}$ est l'involution de l'espace de Hilbert $L^{2}\left(M_{n}\right.$, tr) définie par $J_{n}\left(x_{n} \xi_{n}\right)=$ $x_{n}^{*} \xi_{n}$ si $x_{n}$ est un élément de $M_{n}$.

On notera $\Gamma_{n}$ l'anti-automorphisme de l'algèbre $\mathcal{B}_{n}$ des opérateurs bornés de $L^{2}\left(M_{n}\right.$, tr) défini par:

$$
\Gamma_{n}(v)\left(x \xi_{n}\right)=J_{n} v^{*} J_{n}\left(x \xi_{n}\right) \quad\left(v \in \mathcal{B}_{n}, x \in M_{n}\right) .
$$

On sait que $\Gamma_{n}\left(M_{n}\right)=M_{n}^{\prime}$ et $\Gamma_{n}\left(\pi_{n}(N)^{\prime}\right)=\pi_{n}\left(M_{2 n+1}\right)$ puisque $N \subset M_{n} \subset$ $M_{2 n+1}$ est la construction de base; plus généralement, considérant $M_{p} \subset$ $M_{n} \subset M_{2 n-p}$, on obtient $\Gamma_{n}\left(\pi_{n}\left(M_{p}\right)^{\prime}\right)=\pi_{n}\left(M_{2 n-p}\right)$.

Définition. Soit $A_{k}=N^{\prime} \cap M_{k}$, on note encore tr la restriction à $A_{k}$ de la trace de $M_{k}$. $\Gamma_{n}$ envoie $\pi_{n}\left(A_{2 n+1}\right)$ sur $\pi_{n}\left(A_{2 n+1}\right)$. On appellera $\gamma_{n}$ l'anti-automorphisme de $A_{2 n+1}$ défini par:

$$
\pi_{n}\left(\gamma_{n}(x)\right)=\Gamma_{n}\left(\pi_{n}(x)\right) \quad\left(x \in A_{2 n+1}\right)
$$

Si $0 \leq p \leq n, \gamma_{n}$ coïncide sur $M_{p}^{\prime} \cap M_{2 n-p}$ avec l'anti-automorphisme construit à partir de la tour $M_{p} \subset M_{n} \subset M_{2 n-p}$ (1.5.6).

\subsection{Propriétés fondamentales.}

Théorème 2.2.1. Pour tout $n$ entier naturel, les anti-automorphismes $\gamma_{n}$ satisfont les relations suivantes:

a) $\left.\gamma_{n+2} \gamma_{n+1}\right|_{A_{2 n+1}}=\gamma_{n+1} \gamma_{n}$

b) $f_{n}^{-1} \gamma_{n}(x)=f_{n}^{-1} x \quad\left(x \in A_{n}\right)$

c) $\gamma_{n}\left(e_{k}\right)=e_{2 n-k} \quad(0 \leq k \leq n)$.

La démonstation de ce théorème est l'objet du paragraphe 3. Au cours de cette démonstration, nous donnerons une formule pour $\gamma_{n}(y)$ quand $y$ appartient à $A_{2 n+1}$. 
Proposition 2.2.2 ([Popa $]$ ). Si $N$ est un sous-facteur de profondeur finie dans un facteur $M$ de type $\mathrm{II}_{1}$, les anti-automorphismes $\gamma_{n}$ conservent la trace $\operatorname{tr}$ de $A_{2 n+1}$.

Démonstration. Soit $\ldots N_{p+1} \subset N_{p} \subset \ldots \subset N_{1} \subset N \subset M$ un tunnel dans $N \subset M[\mathbf{G H J}, 4.7 \mathrm{e}] ;$ en considérant la construction de base $N_{p+1} \subset M \subset$ $M_{p+2}$, on peut définir $\gamma_{0}$ un anti-automorphisme de $N_{p+1}^{\prime} \cap M_{p+2}$ par:

$$
\text { si } x \in N_{p+1}^{\prime} \cap M_{p+2} \quad \pi_{0}\left(\gamma_{0}(x)\right)=J_{0}\left(\pi_{0}(x)\right) J_{0}
$$

où $J_{0}$ est l'involution canonique de $L^{2}(M, \operatorname{Tr})$.

Comme $N$ est un sous-facteur de profondeur finie dans un facteur $M$ de type $\mathrm{II}_{1}$, on sait que

$$
E_{N^{\prime} \cap M}\left(e_{0}\right)=[M: N]^{-1} 1 .
$$

Grâce à 4.5 de [PiPo1], 3.1 et 3.2.ii de [PiPo2], cette dernière propriété est suffisante pour affirmer que $\gamma_{0}$ conserve la trace de $N_{p+1}^{\prime} \cap M$ sur $M^{\prime} \cap M_{p+2}$.

La proposition annonce un résultat plus fort. S. Popa affirme que $\gamma_{0}$ conserve la trace de $N_{p+1}^{\prime} \cap M_{p+2}$. Pour démontrer ce résultat, il utilise l'hypotèse de la profondeur finie. En effet, on va plonger $N_{p+1}^{\prime} \cap M_{p+2}$ dans $N_{k+1}^{\prime} \cap M_{k+2}$ tel que $k$ soit supérieur à la profondeur du graphe principal. $\gamma_{0}$ s'étend à $N_{k+1}^{\prime} \cap M_{k+2}$ et conserve la trace de $N_{k+1}^{\prime} \cap M$ sur $M^{\prime} \cap M_{k+2}$. Ce choix de $k$ permet d'affirmer que $N_{k+1}^{\prime} \cap M_{1}$ est l'espace vectoriel engendré par $N_{k+1}^{\prime} \cap M e_{0} N_{k+1}^{\prime} \cap M$ donc $\gamma_{0}$ conserve la trace de $N_{k+1}^{\prime} \cap M_{1}$ sur $N^{\prime} \cap M_{p+2}$; par récurrence, on montre ainsi que $\gamma_{0}$ conserve la trace de $N_{p+1}^{\prime} \cap M_{p+2}$.

De la même façon, $\gamma_{n}$ conserve la trace sur $A_{2 n+1}$, il suffit, pour le démontrer, d'opérer une translation des indices sur la suite d'algèbres formée du tunnel et de la tour dérivée.

Remarque. La relation (a) du théorème 2.2 .1 permet de définir un isomorphisme $T$ de la tour dérivée de l'inclusion $N \subset M$ sur la tour dérivée de $M_{1} \subset M_{3}$ :

Soient $A_{p}=N^{\prime} \cap M_{p}$ et $B_{p}=M_{1}^{\prime} \cap M_{p}$,

$T$ est égal à $\gamma_{n+1} \gamma_{n}$ sur $A_{2 n+1}$ et $T\left(A_{2 n+1}\right)=B_{2 n+1}$.

D'après (2.2.1a), $T$ est bien défini, c'est un isomorphisme des tours dérivées conservant la trace (2.2.2) et les anti-isomorphismes, c'est un isomorphisme de paragroupe (voir 4) qui opère une translation de 2 sur les indices des projecteurs de V. Jones. 


\section{Démonstration du théorème 2.2.1.}

\subsection{Lemmes.}

\section{Lemme 3.1.1.}

a)

$$
\begin{aligned}
& f_{n}^{p}=\alpha^{(n-p-1)} g_{n}^{p+1} f_{n+1}^{p+2}\left(g_{n}^{p+1}\right)^{*} \text { et } \\
& \pi_{n+1}\left(f_{n}^{p}\right)=\alpha^{(n-p-1)} g_{n}^{p+1} \pi_{n+1}\left(f_{n+1}^{p+2}\right)\left(g_{n}^{p+1}\right)^{*}
\end{aligned}
$$

$\left(g_{n}^{p+1}\right.$, élément de $M_{n+1}$, agit par multiplication à gauche sur $\left.L^{2}\left(M_{n+1}, \operatorname{tr}\right)\right)$.

b) $f_{n+1}^{-1}=\alpha^{n+1} g_{n+1}^{2 n+2} f_{n}^{-1} g_{2 n+2}^{n+1}$.

Démonstration.

a)

$$
\begin{aligned}
f_{n}^{p} & =\alpha(n, p) g_{n}^{p+1} g_{n+1}^{p+2} \ldots g_{2 n-p-1}^{n} \\
& =\alpha(n, p) g_{n}^{p+1} e_{p+1} g_{n+1}^{p+3} e_{p+2} \ldots g_{n+k-1}^{p+k+1} e_{p+k} \ldots g_{2 n-p-1}^{n+1} e_{n}
\end{aligned}
$$

Grâce aux règles de commutation des projecteurs (1.5.3c), on obtient:

$$
f_{n}^{p}=\alpha(n, p) g_{n}^{p+1} g_{n+1}^{p+3} \ldots g_{n+k-1}^{p+k+1} \ldots g_{2 n-p-1}^{n+1} e_{p+1} e_{p+2} \ldots e_{p+k} \ldots e_{n}
$$

Comme $f_{n+1}^{p+2}=\alpha(n+1, p+2) g_{n+1}^{p+3} \ldots g_{n+k-1}^{p+k+1} \ldots g_{2 n-p-1}^{n+1}$ et $\alpha(p, n)=\alpha^{(n-p-1)} \alpha(n+1, p+2), f_{n}^{p}$ vaut $\alpha^{(n-p-1)} g_{n}^{p+1} f_{n+1}^{p+2}\left(g_{n}^{p+1}\right)^{*}$ et (a) est démontré.

b)

$$
\begin{aligned}
f_{n+1}^{-1} & =\alpha(n+1,-1) g_{n+1}^{0} g_{n+2}^{1} \ldots g_{2 n+2}^{n+1} \\
& =\alpha(n+1,-1) e_{n+1} g_{n}^{0} e_{n+2} g_{n+1}^{1} \ldots e_{2 n+1} g_{2 n}^{n} e_{2 n+2} g_{2 n+2}^{n+1} \\
& =\alpha(n+1,-1) g_{n+1}^{2 n+2} g_{n}^{0} g_{n+1}^{1} \ldots g_{2 n}^{n} g_{2 n+2}^{n+1} \\
& =\alpha(n,-1) \alpha^{n+1} g_{n+1}^{2 n+2}\left(g_{n}^{0} g_{n+1}^{1} \ldots g_{2 n}^{n}\right) g_{2 n+2}^{n+1} \\
& =\alpha^{n+1} g_{n+1}^{2 n+2} f_{n}^{-1} g_{2 n+2}^{n+1}
\end{aligned}
$$

Lemme 3.1.2. (dit du tour de passe-passe)

$$
g_{k+3}^{k+1} e_{k+3}=e_{k+1} g_{k+3}^{k+1}
$$


Démonstration.

$$
\begin{aligned}
g_{k+3}^{k+1} e_{k+3} & =\left(e_{k+3} e_{k+2} e_{k+1}\right) e_{k+3} \\
& =\left(e_{k+3} e_{k+2} e_{k+3}\right) e_{k+1} \\
& =e_{k+3}\left(\alpha e_{k+1}\right) \\
& =e_{k+3}\left(e_{k+1} e_{k+2} e_{k+1}\right) \\
& =e_{k+1}\left(e_{k+3} e_{k+2} e_{k+1}\right)
\end{aligned}
$$

Lemme 3.1.3. Si $0 \leq k \leq n, e_{2 n-k} f_{n}^{-1}=e_{k} f_{n}^{-1}$.

Démonstration.

$$
\begin{aligned}
e_{2 n-k} f_{n}^{-1} & =e_{2 n-k} \alpha(n,-1) g_{n}^{0} g_{n+1}^{1} \ldots g_{2 n}^{n} \\
& =\alpha(n,-1) g_{n}^{0} g_{n+1}^{1} \ldots g_{2 n-k-2}^{n-k-2} e_{2 n-k} g_{2 n-k-1}^{n-k-1} \ldots g_{2 n}^{n}
\end{aligned}
$$

Nous avons $e_{2 n-k}$ devant $g_{2 n-k-1}^{n-k-1}$ et nous voudrions $e_{k}$ devant $g_{n}^{0}$.

$1^{\text {ère }}$ étape: transformons $e_{2 n-k}$ en $e_{2 n-k-2}$ devant $g_{2 n-k-1}^{n-k-1}$. D'abord nous faisons disparaître $e_{2 n-k}$ :

$$
e_{2 n-k} g_{2 n-k-1}^{n-k-1} g_{2 n-k}^{n-k}=e_{2 n-k} e_{2 n-k-1} g_{2 n-k-2}^{n-k-1} e_{2 n-k} g_{2 n-k-1}^{n-k}
$$

Comme $e_{2 n-k}$ et $g_{2 n-k-2}^{n-k-1}$ commutent [(1.5.3a) et (1.5.3b)],

$$
\begin{aligned}
e_{2 n-k} g_{2 n-k-1}^{n-k-1} g_{2 n-k}^{n-k} & =e_{2 n-k} e_{2 n-k-1} e_{2 n-k} g_{2 n-k-2}^{n-k-1} g_{2 n-k-1}^{n-k} \\
& =\alpha g_{2 n-k-2}^{n-k-1} e_{2 n-k} g_{2 n-k-1}^{n-k} \\
& =\alpha g_{2 n-k-2}^{n-k-1} g_{2 n-k}^{n-k} .
\end{aligned}
$$

Ensuite nous faisons apparaître $e_{2 n-k-2}$ :

$$
\begin{aligned}
e_{2 n-k} g_{2 n-k-1}^{n-k-1} g_{2 n-k}^{n-k} & =\alpha e_{2 n-k-2} g_{2 n-k-3}^{n-k-1} g_{2 n-k}^{n-k} \\
& =e_{2 n-k-2} e_{2 n-k-1} e_{2 n-k-2} g_{2 n-k-3}^{n-k-1} g_{2 n-k}^{n-k} \\
& =e_{2 n-k-2} g_{2 n-k-1}^{n-k-1} g_{2 n-k}^{n-k} \quad(1.5 .1 \mathrm{c})
\end{aligned}
$$

$2^{\grave{e ̀ m e}}$ étape: nous avons maintenant $e_{2 n-k-2}$ entre $g_{2 n-k-2}^{n-k-2}$ et $g_{2 n-k-1}^{n-k-1}$, grâce à $n-k-1$ tours de passe-passe (3.1.2), nous allons obtenir $e_{k}$ devant $g_{n}^{0}$ :

$1^{\text {er }}$ tour de passe-passe:

$$
\begin{aligned}
g_{2 n-k-2}^{n-k-2} e_{2 n-k-2} & =e_{2 n-k-2} e_{2 n-k-3} e_{2 n-k-4} e_{2 n-k-2} g_{2 n-k-5}^{n-k-2} \\
& =e_{2 n-k-4} e_{2 n-k-2} e_{2 n-k-3} e_{2 n-k-4} g_{2 n-k-5}^{n-k-2} \\
& =e_{2 n-k-4} g_{2 n-k-2}^{n-k-2} .
\end{aligned}
$$


Après $p$ tours de passe-passe, nous avons $e_{2 n-k-2(p+1)}$ devant $g_{2 n-k-(p+1)}^{n-k-(p+1)}$ et après $n-k-1$ tours de passe-passe, nous obtenons $e_{k}$ devant $g_{n}^{0}$ et la relation annoncée est vérifiée.

Lemme 3.1.4. Si $x \in A_{n}, x_{n} \in M_{n}, \pi_{n}\left(\gamma_{n}(x)\right)\left(x_{n} \xi_{n}\right)=x_{n} x \xi_{n}$.

Démonstration.

$$
\pi_{n}\left(\gamma_{n}(x)\right)\left(x_{n} \xi_{n}\right)=J_{n} \pi_{n}(x)^{*} J_{n}\left(x_{n} \xi_{n}\right)=J_{n} \pi_{n}(x)^{*}\left(x_{n}^{*} \xi_{n}\right) .
$$

Comme $x$ est un élément de $M_{n}, \pi_{n}\left(\gamma_{n}(x)\right)\left(x_{n} \xi_{n}\right)$ est donc égal à $J_{n}\left(x^{*} x_{n}^{*} \xi_{n}\right)$, soit $x_{n} x \xi_{n}$. Ainsi $\gamma_{n}(x)$, élément de $M_{n}^{\prime}$, agit par multiplication à droite sur $L^{2}(M, \operatorname{tr})$.

Lemme 3.1.5. Si la famille $\left\{m_{j}, 1 \leq j \leq a_{n}+1\right\}$ est une base de PimsnerPopa de $M_{n-1}$ sur $N$, alors la famille $\left\{\alpha^{n / 2} \lambda_{i} g_{0}^{n-1} m_{j}, 1 \leq j \leq a_{n}+1,1 \leq\right.$ $i \leq a+1\}$ est une base de Pimsner-Popa de $M_{n}$ sur $N$.

Démonstration. Vérifions (a) est (b) de (1.5.2):

$$
\begin{aligned}
E_{N}\left(\left(\alpha^{n / 2} m_{h}^{*} g_{n-1}^{0} \lambda_{k}^{*}\right)\right. & \left.\left(\alpha^{n / 2} \lambda_{i} g_{0}^{n-1} m_{j}\right)\right)=\alpha^{n} E_{N}\left(m_{h}^{*} g_{n-1}^{1} e_{0}\left(\lambda_{k}^{*} \lambda_{i}\right) e_{0} g_{1}^{n-1} m_{j}\right) \\
& =\alpha^{n} \delta_{i, k} E_{N}\left(m_{h}^{*} g_{n-1}^{1} e_{0} g_{1}^{n-1} m_{j}\right) \quad(1.5 .1 \mathrm{c}, \mathrm{d} \text { et } 3 \mathrm{~d}) \\
& =\alpha^{1} \delta_{i, k} E_{N}\left(m_{h}^{*} E_{M_{n-1}}\left(e_{n-1}\right) m_{j}\right)=\delta_{i, k} \delta_{j, h}(1.5 .2 \mathrm{a}) .
\end{aligned}
$$

Quand l'indice $\alpha$ de $N$ dans $M$ est entier, on peut facilement donner une base de Pimsner-Popa de $M_{n}$ sur $N$ :

Proposition 3.1.6. On suppose que l'indice $\alpha$ de $N$ dans $M$ est entier.

On note $\mathcal{J}_{n}$ le produit de $n$ copies de $\{0,1,2, \ldots, \alpha\}$ et si $I=\left(i_{0}, i_{1}\right.$, $\left.i_{2}, \ldots, i_{n}\right)$ est un élément de $\mathcal{J}_{n+1}$, on pose

$$
m_{I}=\alpha^{n(n+1) / 4} \lambda_{i_{n}} g_{0}^{n-1} \lambda_{i_{n-1}} g_{0}^{n-2} \ldots \lambda_{i_{2}} g_{0}^{1} \lambda_{i_{1}} e_{0} \lambda_{i_{0}} .
$$

Alors la famille $\left\{m_{I}, I \in \mathcal{J}_{n+1}\right\}$ est une base de Pimsner-Popa de $M_{n}$ sur $N$.

Ceci résulte par récurrence de (1.5.2g) et du Lemme 3.1.5.

3.2. Une formule pour $\gamma_{n}(y)$ quand $y$ appartient à $A_{2 n+1}$. Nous allons maintenant donner l'expression de $\gamma_{n}(y)$ quand $y$ appartient à $A_{2 n+1}$. Cette formule nous permettra d'établir 2.2.1(a) et nous sera très utile dans la partie 5. Quand l'indice est entier, elle cö̈ncide, grâce à la proposition 3.1.6, avec celle annoncée par A. Ocneanu. 
Proposition 3.2.1. Si $\left\{m_{i}, 1 \leq i \leq a_{n+1}+1\right\}$ est une base de Pimsner-Popa de $M_{n}$ sur $N$, alors pour tout $y$ de $A_{2 n+1}$, on a:

$$
\gamma_{n}(y)=\alpha^{n+1} \sum_{k=1}^{a_{n+1}+1} E_{M_{n}}\left(f_{n}^{-1} m_{k} y\right) f_{n}^{-1} m_{k}^{*} .
$$

Démonstration. D'après 1.5.2, la famille $\left\{\alpha^{(n+1) / 2} m_{i} f_{n}^{-1}, 1 \leq i \leq a_{n+1}+1\right\}$ est une base de Pimsner-Popa de $M_{2 n+1}$ sur $M_{n}$ et

$$
y=\alpha^{2 n+1} \sum_{i=1}^{a_{n+1}+1} E_{M_{n}}\left(y m_{i} f_{n}^{-1}\right) f_{n}^{-1} m_{i}^{*} \text {. }
$$

Si $E_{N}\left(m_{h}^{*} m_{h}\right)=q_{h}$, tout élément de $M_{n}$ s'écrit $\sum_{k+1}^{a_{n+1}+1} z_{h} m_{h}^{*}$ où $z_{h}$ est un élément de $N q_{h}$, il suffit donc de connaître $\pi_{n}\left(\gamma_{n}(y)\right)$ sur les vecteurs de $L^{2}\left(M_{n}, \operatorname{tr}\right)$ de la forme $z m_{h}^{*} \xi_{n}$ où $z$ appartient à $N_{q_{h}}$. J'omets $\pi_{n}$ quand l'élément de $M_{n}$ agit par multiplication à gauche.

$$
\begin{aligned}
\pi_{n}\left(y^{*}\right) J_{n}\left(z m_{h}^{*} \xi_{n}\right) & =\alpha^{n+1} \sum_{i=1}^{a_{n+1}+1} E_{M_{n}}\left(y^{*} m_{i} f_{n}^{-1}\right) \pi_{n}\left(f_{n}^{-1}\right)\left(m_{i}^{*} m_{h} z^{*} \xi_{n}\right) \\
& =\alpha^{n+1} \sum_{i=1}^{a_{n}+1+1} E_{M_{n}}\left(y^{*} m_{i} f_{n}^{-1}\right) E_{N}\left(m_{i}^{*} m_{h}\right)\left(z^{*} \xi_{n}\right) .
\end{aligned}
$$

Or

$$
E_{N}\left(m_{i}^{*} m_{h}\right) z^{*}=\delta_{i, h} q_{h} z^{*}=\delta_{i, h} z^{*} \quad(1.5 .2 \mathrm{a}, \mathrm{b}),
$$

d'où

$$
\pi_{n}\left(y^{*}\right) J_{n}\left(z m_{h}^{*} \xi_{n}\right)=\alpha^{n+1} E_{M_{n}}\left(y^{*} m_{h} f_{n}^{-1}\right)\left(z^{*} \xi_{n}\right)
$$

et

$$
\pi_{n}\left(\gamma_{n}(y)\right)\left(z m_{h}^{*} \xi_{n}\right)=\alpha^{n+1} z E_{M_{n}}\left(f_{n}^{-1} m_{h}^{*} y\right) \xi_{n} .
$$

Comme $z$ commute à $E_{M_{n}}$ et $f_{n}^{-1}$ et que $z m_{h}^{*}$ se décompose en

$$
\sum_{k=1}^{a_{n+1}+1} m_{k} E_{N}\left(m_{k}^{*} z m_{h}^{*}\right)
$$

on obtient:

$$
\pi_{n}\left(\gamma_{n}(y)\right)\left(z m_{h}^{*} \xi_{n}\right)=\alpha^{n+1} \sum_{k=1}^{a_{n+1}+1} E_{M_{n}}\left(f_{n}^{-1} m_{k} E_{N}\left(m_{k}^{*} z m_{h}^{*}\right) y\right) \xi_{n} .
$$


Comme l'élément $y$ de $A_{2 n+1}$ commute à $E_{N}\left(m_{k}^{*} z m_{h}^{*}\right)$, on obtient:

$$
\begin{array}{r}
\pi_{n}\left(\gamma_{n}(y)\right)\left(z m_{h}^{*} \xi_{n}\right)=\alpha^{n+1} \sum_{k=1}^{a_{n+1}+1} E_{M_{n}}\left(f_{n}^{-1} m_{k} y\right) E_{N}\left(m_{k}^{*} z m_{h}^{*}\right) \xi_{n} \\
=\alpha^{n+1} \sum_{k=1}^{a_{n+1}+1} E_{M_{n}}\left(f_{n}^{-1} m_{k} y\right) \pi_{n}\left(f_{n}^{-1}\right) m_{k}^{*}\left(z m_{h}^{*} \xi_{n}\right) .
\end{array}
$$

Et la formule est démontrée.

3.3. $\left.\gamma_{n+2} \gamma_{n+1}\right|_{A_{2 n+1}}=\gamma_{n+1} \gamma_{n}$.

Lemme 3.3.1. Si $x \in M_{n}, E_{M_{1}}\left(g_{1}^{n} x g_{n}^{1}\right)=\alpha^{-n} E_{M}(x)$.

Démonstration.

$$
\begin{aligned}
E_{M_{1}}\left(g_{1}^{n} x g_{n}^{1}\right) & =E_{M_{1}}\left(e_{1} e_{2} \ldots e_{n} x e_{n} \ldots e_{2} e_{1}\right) \\
& =E_{M_{1}}\left(e_{1} e_{2} \ldots e_{n-1} E_{M_{n-1}}(x) e_{n} e_{n-1} \ldots e_{2} e_{1}\right) .
\end{aligned}
$$

Comme $E_{M_{1}}$ est égal à $E_{M_{1}} E_{M_{n}}$, l'égalité devient:

$$
E_{M_{1}}\left(g_{1}^{n} x g_{n}^{1}\right)=\alpha^{-1} E_{M_{1}}\left(e_{1} e_{2} \ldots e_{n-1} E_{M_{n-1}}(x) e_{n-1} \ldots e_{2} e_{1}\right) .
$$

Après $n-1$ manœuvres de ce type, on obtient:

$$
E_{M_{1}}\left(g_{1}^{n} x g_{n}^{1}\right)=\alpha^{-(n-1)} E_{M_{1}}\left(e_{1} E_{M_{1}}(x) e_{1}\right)=\alpha^{-n} E_{M}(x) \text {. }
$$

Proposition 3.3.2. Si, pour $1 \leq k \leq a+1, \mu_{k}=\alpha^{(2 n+2) / 2} \lambda_{k} g_{0}^{2 n+1}$ et si $y$ est un élément de $A_{2 n+1}$, alors $\gamma_{n+1}(y)$ est l'élément $\sum_{k=1}^{a+1} \mu_{k} \gamma_{n}(y) e_{2 n+2} \mu_{k}^{*}$ de $M_{1}^{\prime} \cap M_{2 n+3}$.

Démonstration. Comme $\left\{\alpha^{(n+1) / 2} \lambda_{i} g_{0}^{n}, i=1, \ldots, n\right\}$ est une base de PimsnerPopa de $M_{n+1}$ sur $M_{n}(1.5 .2)$, il suffit de calculer $\pi_{n+1}\left(\gamma_{n+1}(y)\right)$ sur les vecteurs de $L^{2}\left(M_{n+1}\right.$, tr) de la forme $\lambda_{j} g_{0}^{n} z \xi_{n+1}$ où $p_{j}$ est le projecteur $E_{N}\left(\lambda_{j}^{*} \lambda_{j}\right)$ et $z$ un élément de $p_{j} M_{n}$.

$$
\begin{aligned}
& \pi_{n+1}\left(y^{*}\right) J_{n+1}\left(\lambda_{j} g_{0}^{n} z \xi_{n+1}\right) \\
& =\alpha^{2 n+1} \sum_{i=1}^{a_{n+1}+1} E_{M_{n}}\left(y^{*} m_{i} f_{n}^{-1}\right) \pi_{n+1}\left(f_{n}^{-1}\right)\left(m_{i}^{*} z^{*} g_{n}^{0} \lambda_{j}^{*} \xi_{n+1}\right) .
\end{aligned}
$$

Comme $f_{n}^{-1}=\alpha^{n} g_{n}^{0} f_{n+1}^{1}\left(g_{n}\right)^{*}$ et $\pi_{n+1}\left(f_{n+1}^{1}\right)=E_{M_{1}}(1.5 .3 \mathrm{~h})$, on a:

$$
\begin{aligned}
& \pi_{n+1}\left(y^{*}\right) J_{n+1}\left(\lambda_{j} g_{0}^{n} z \xi_{n+1}\right) \\
& \quad=\alpha^{2 n+1} \sum_{i=1}^{a_{n+1}+1} E_{M_{n}}\left(y^{*} m_{i} f_{n}^{-1}\right) g_{n}^{0} E_{M_{1}}\left(g_{0}^{n} m_{i}^{*} z^{*} g_{n}^{0} \lambda_{j}^{*}\right) \xi_{n+1} .
\end{aligned}
$$




\section{Donc}

$$
\begin{aligned}
\pi_{n+1}\left(\gamma_{n+1}(y)\right) & \left(\lambda_{j} g_{0}^{n} z \xi_{n+1}\right) \\
& =\alpha^{2 n+1} \sum_{i=1}^{a_{n+1}+1} E_{M_{1}}\left(\lambda_{j} g_{0}^{n} z m_{i} g_{n}^{0}\right) g_{0}^{n} E_{M_{n}}\left(f_{n}^{-1} m_{i}^{*} y\right) \xi_{n+1} \\
& =\alpha^{2 n+1} \sum_{i=1}^{a_{n+1}+1} \lambda_{j} e_{0} E_{M_{1}}\left(g_{1}^{n} z m_{i} g_{n}^{1}\right) g_{0}^{n} E_{M_{n}}\left(f_{n}^{-1} m_{i}^{*} y\right) \xi_{n+1} .
\end{aligned}
$$

Appliquons le lemme 3.3.1 à $z m_{i}$ et nous obtenons:

$$
\begin{aligned}
\pi_{n+1}\left(\gamma_{n+1}(y)\right)\left(\lambda_{j} g_{0}^{n} z \xi_{n+1}\right) & \\
& =\alpha^{n+1} \sum_{i=1}^{a_{n+1}+1} \lambda_{j} e_{0} E_{M}\left(z m_{i}\right) e_{0} g_{o}^{n} E_{M_{n}}\left(f_{n}^{-1} m_{i}^{*} y\right) \xi_{n+1} \\
& =\alpha^{n+1} \sum_{i=1}^{a_{n+1}+1} \lambda_{j} e_{0} E_{N}\left(z m_{i}\right) g_{0}^{n} E_{M_{n}}\left(f_{n}^{-1} m_{i}^{*} y\right) \xi_{n+1}
\end{aligned}
$$

Comme $E_{N}\left(z m_{i}\right)$ commute à $g_{0}^{n}$, à $E_{M_{n}}$ et à $f_{n}^{-1}$ et que $\sum_{i=1}^{a_{n+1}+1} E_{N}\left(z m_{i}\right) m_{i}^{*}$ vaut $z$, on peut écrire:

$$
\pi_{n+1}\left(\gamma_{n+1}(y)\right)\left(\lambda_{j} g_{0}^{n} z \xi_{n+1}\right)=\alpha^{n+1} \lambda_{j} g_{0}^{n} E_{M_{n}}\left(f_{n}^{-1} z y\right) \xi_{n+1} .
$$

On utilise maintenant la décomposition de $\xi$ sur la base $m_{i}$ (1.5.2f):

$$
z=\sum_{i=1}^{a_{n+1}+1} m_{i} E_{N}\left(m_{i}^{*} z\right)
$$

pour faire commuter $z$ avec $y$, élément de $A_{2 n+1}$ et le sortir de $E_{M_{n}}$ :

$$
\begin{array}{r}
\pi_{n+1}\left(\gamma_{n+1}(y)\right)\left(\lambda_{j} g_{0}^{n} z \xi_{n+1}\right)=\alpha^{n+1} \sum_{i=1}^{a_{n}+1} \lambda_{j} g_{0}^{n} E_{M_{n}}\left(f_{n}^{-1} m_{i} y\right) E_{N}\left(m_{i}^{*} z\right) \xi_{n+1} \\
=\alpha^{n+1} \sum_{i=1, k=1}^{a_{n+1}+1, a+1} \delta_{k, j} \lambda_{k} g_{o}^{n} E_{M_{n}}\left(f_{n}^{-1} m_{i} y\right) E_{N}\left(m_{i}^{*} p_{j} z\right) \xi_{n+1} .
\end{array}
$$

Comme $\delta_{k, j} p_{j}=\alpha^{(n+1)} E_{M_{n}}\left(g_{n}^{0} \lambda_{k}^{*} \lambda_{j} g_{0}^{n}\right), \pi_{n+1}\left(\gamma_{n+1}(y)\right)\left(\lambda_{j} g_{0}^{n} z \xi_{n+1}\right)$ est égal à

$$
\begin{aligned}
& \alpha^{2(n+1)} \sum_{i=1, k=1}^{a_{n+1}+1, a+1} \lambda_{k} g_{0}^{n} E_{M_{n}}\left(f_{n}^{-1} m_{i} y\right) E_{N}\left(m_{i}^{*} E_{M_{n}}\left(g_{n}^{0} \lambda_{k}^{*} \lambda_{j} g_{0}^{n}\right) z\right) \xi_{n+1} . \\
& \pi_{n+1}\left(\gamma_{n+1}(y)\right)\left(\lambda_{j} g_{0}^{n} z \xi_{n+1}\right) \\
& =\alpha^{2(n+1)} \sum_{i=1, k=1}^{a_{n+1}+1, a+1} \lambda_{k} g_{0}^{n} E_{M_{n}}\left(f_{n}^{-1} m_{i} y\right) E_{N}\left(m_{i}^{*} g_{n}^{0} \lambda_{k}^{*} \lambda_{j} g_{0}^{n} z\right) \xi_{n+1} .
\end{aligned}
$$


Or $\pi_{n+1}\left(f_{n+1}^{-1}\right)(x) \xi_{n+1}=E_{N}(x) \xi_{n+1}$ si $x \in M_{n+1}(1.5 .3 \mathrm{~h})$, donc

$$
\gamma_{n+1}(y)=\alpha^{2(n+1)} \sum_{i=1, k=1}^{a_{n}+1, a+1} \lambda_{k} g_{0}^{n} E_{M_{n}}\left(f_{n}^{-1} m_{i} y\right) f_{n+1}^{-1} m_{i}^{*} g_{n}^{0} \lambda_{k}^{*}
$$

Comme $f_{n+1}^{-1}$ est égal à $\alpha^{n+1} g_{n+1}^{2 n+2} f_{n}^{-1} g_{2 n+2}^{n+1}$ (3.1.1), en utilisant les règles de commutation, on écrit:

$$
\gamma_{n+1}(y)=\alpha^{3(n+1)} \sum_{k=1}^{a+1} \lambda_{k} g_{0}^{2 n+2}\left[\sum_{i=1}^{a_{n+1}+1} E_{M_{n}}\left(f_{n}^{-1} m_{i} y\right) f_{n}^{-1} m_{i}^{*}\right] g_{2 n+2}^{0} \lambda_{k}^{*}
$$

D'où $\gamma_{n+1}(y)=\alpha^{2(n+1)} \sum_{k=1}^{a+1} \lambda_{k} g_{0}^{2 n+2} \gamma_{n}(y) g_{2 n+2}^{0} \lambda_{k}^{*}$.

$\mathrm{Si}$, pour $1 \leq k \leq a+1$, on note $\mu_{k}$ l'élément $\alpha^{2(n+1) / 2} \lambda_{k} g_{0}^{n+1}$ de la base de $M_{2 n+2}$ sur $M_{2 n+1}$, ce résultat s'écrit:

$$
\gamma_{n+1}(y)=\sum_{k=1}^{a+1} \mu_{k} \gamma_{n}(y) e_{2 n+2} \mu_{k}^{*}
$$

Corollaire 3.3.3. Si, pour $1 \leq k \leq a+1, \mu_{k}=\alpha^{(2 n+2) / 2} \lambda_{k} g_{0}^{2 n+1}$ et si $y$ est un élément de $A_{2 n+1}$, alors

$$
\gamma_{n+1}\left(\gamma_{n}(y)\right)=\sum_{k=1}^{a+1} \mu_{k} y e_{2 n+2} \mu_{k}^{*}
$$

Proposition 3.3.4. $\left.\gamma_{n+2} \gamma_{n+1}\right|_{A_{2 n+1}}=\gamma_{n+1} \gamma_{n} \quad(n \in \mathbb{N})$.

Démonstration. Ecrivons la formule du corollaire 3.3.3 pour $n+1$ : Si $y$ est un élément de $A_{2 n+3}$,

$$
\gamma_{n+2}\left(\gamma_{n+1}(y)\right)=\alpha^{2} \sum_{k=1}^{a+1} \mu_{k} e_{2 n+2} e_{2 n+3} y e_{2 n+4} e_{2 n+3} e_{2 n+2} \mu_{k}^{*}
$$

Mais si, de plus, $y$ est dans $A_{2 n+1}$, la formule se simplifie car

$$
\begin{aligned}
\alpha^{2} e_{2 n+2} e_{2 n+3} y e_{2 n+4} e_{2 n+3} e_{2 n+2} & =\alpha^{2} y e_{2 n+2}\left(e_{2 n+3} e_{2 n+4} e_{2 n+3}\right) e_{2 n+2} \\
& =\alpha y\left(e_{2 n+2} e_{2 n+3} e_{2 n+2}\right)=y
\end{aligned}
$$

On obtient alors:

$$
\gamma_{n+2}\left(\gamma_{n+1}(y)\right)=\gamma_{n+1}\left(\gamma_{n}(y)\right)
$$


3.4. $f_{n}^{-1} \gamma_{n}(x)$ et $f_{n}^{-1}$ sont égaux si $x$ est un élément de $A_{n}$.

Proposition 3.4. Pour tout $x$ de $A_{n}, f_{n}^{-1} \gamma_{n}(x)=f_{n}^{-1} x$.

Démonstration. Il suffit de démontrer la relation pour les unitaires de $A_{n}$. Soient $u$ un unitaire de $A_{n}$ et $z$ un élément de $M_{n}$,

$$
\pi_{n}\left(f_{n}^{-1} \gamma_{n}(u)\right)\left(z \xi_{n}\right)=\pi_{n}\left(f_{n}^{-1}\right)\left(z u \xi_{n}\right)
$$

Alors par définition de $f_{n}^{-1}, \pi_{n}\left(f_{n}^{-1} \gamma_{n}(u)\right)\left(z \xi_{n}\right)=E_{N}(z u) \xi_{n}$. Comme $u$ normalise $N$, pour tout $x$ de $M_{n}, E_{N}\left(u x u^{*}\right)=u E_{N}(x) u^{*}$, on obtient donc:

$$
\pi_{n}\left(f_{n}^{-1} \gamma_{n}(u)\right)\left(z \xi_{n}\right)=E_{N}(z u) \xi_{n}=u^{*} E_{N}(u z) u \xi_{n}=E_{N}(u z) \xi_{n}
$$

car $u$ commute à $N$. Cela s'écrit aussi:

$$
\pi_{n}\left(f_{n}^{-1} \gamma_{n}(u)\right)\left(z \xi_{n}\right)=\pi_{n}\left(f_{n}^{-1} u\right)\left(z \xi_{n}\right) .
$$

La relation est démontrée.

3.5. Si $0 \leq k \leq n$, l'image de $e_{k}$ par l'anti-automorphisme $\gamma_{n}$ est $e_{2 n-k}$.

Proposition 3.5. $\gamma_{n}\left(e_{k}\right)=e_{2 n-k} \quad(0 \leq k \leq n)$.

Démonstration.

$1^{\text {er }}$ cas: $k=n$.

$e_{n}$ est la projection de $L^{2}\left(M_{n}, \operatorname{tr}\right)$ sur $L^{2}\left(M_{n-1}\right.$, tr $)$, aussi $J_{n}$ et $e_{n}$ commutent et $\gamma_{n}\left(e_{n}\right)=e_{n}$.

$2^{\text {ème }}$ cas: $0 \leq k<n$.

Soient $x, y, z$ des éléments de $M_{n}$. Calculons d'abord $\gamma_{n}\left(e_{k}\right) x f_{n}^{-1} y$ :

$\pi_{n}\left(\gamma_{n}\left(e_{k}\right) x f_{n}^{-1} y\right)\left(z \xi_{n}\right)=\pi_{n}\left(\gamma_{n}\left(e_{k}\right)\right)\left(x E_{N}(y z) \xi_{n}\right)=x E_{N}(y z) e_{k} \xi_{n}$

Comme $e_{k}$ commute à $M_{k-1}$ donc à $N$,

$$
\pi_{n}\left(\gamma_{n}\left(e_{k}\right) x f_{n}^{-1} y\right)\left(z \xi_{n}\right)=x e_{k} E_{N}(y z) \xi_{n} .
$$

On obtient donc $\gamma_{n}\left(e_{k}\right) x f_{n}^{-1} y=x e_{k} f_{n}^{-1} y$.

Calculons maintenant $e_{2 n-k} x f_{n}^{-1} y$ :

Comme $k<n, e_{2 n-k}$ commute à $M_{n}$, donc

$$
e_{2 n-k} x f_{n}^{-1} y=x e_{2 n-k} f_{n}^{-1} y=x e_{k} f_{n}^{-1} y
$$

Soient $a_{n+1}$ la partie entière de $\alpha^{n+1}$, l'indice de $N$ dans $M_{n}$ et

$$
\left\{m_{\jmath}, j=1, \ldots a_{n+1}+1\right\}
$$


une base de Pimsner-Popa de $M_{n}$ sur $N$. Comme $\sum_{j=1}^{a_{n+1}+1} m_{j} f_{n}^{-1} m_{j}^{*}$ est l'identité (1.5.2d) et, pour tous $x$ et $y$ éléments de $M_{n}, \gamma_{n}\left(e_{k}\right) x f_{n}^{-1} y$ et $e_{2 n-k} x f_{n}^{-1} y$ coïncident, on peut écrire:

$$
\gamma_{n}\left(e_{k}\right)\left(\sum_{j=1}^{a_{n+1}+1} m_{j} f_{n}^{-1} m_{j}^{*}\right)=e_{2 n-k}\left(\sum_{j=1}^{a_{n+1}+1} m_{j} f_{n}^{-1} m_{j}^{*}\right)
$$

c'est-à-dire $\gamma_{n}\left(e_{k}\right)=e_{2 n-k}$.

\section{Le paragroupe, invariant complet pour l'inclusion d'un sous-facteur de profondeur finie dans le facteur hyperfini de type $\mathrm{II}_{1}$.}

4.1. Paragroupe ou carré commutatif. Popa a montré que l'inclusion d'un sous-facteur de profondeur finie dans le facteur hyperfini de type $\mathrm{II}_{1}$ est determinée par son carré commutatif canonique [Popa1, 6.6]. Le paragroupe est une autre version de cet invariant.

Définition. Le paragroupe de l'inclusion $N \subset M$ est la tour dérivée $\left(A_{k}\right)_{k \geq 0}$ de $N \subset M$ munie de ses anti-automorphismes canoniques $\gamma_{k}$.

Remarque. La donnée du graphe principal équivaut à celle de la tour dérivée [GHJ, 4.6.5].

Théorème 4.1.1. Soient $N$ (resp. $\tilde{N})$ un sous-facteur de profondeur finie dans le facteur hyperfini de type $\mathrm{II}_{1} M$ (resp. $\left.\widetilde{M}\right)$. Si les couples $N \subset M$ et $\tilde{N} \subset \widetilde{M}$ ont même paragroupe, ils sont isomorphes.

Démonstration. On suppose qu'il existe un isomorphisme $\beta$ des tours dérivées conservant la trace tel que $\beta\left(A_{k}\right)=\widetilde{A}_{k}$ et $\gamma_{k}=\beta^{-1} \widetilde{\gamma}_{k} \beta$.

Si $N \subset M$ est de profondeur finie $p, N_{1} \subset N$ est de profondeur finie $p_{1}$, alors si $2 j$ est supérieur à $p-1$ et $p_{1}$, le carré commutatif

$$
\begin{array}{ccc}
M^{\prime} \cap M_{2 j} & \subset N^{\prime} \cap M_{2 j} \\
\cap & \cap & \cap \\
M^{\prime} \cap M_{2 j+1} & \subset N^{\prime} \cap M_{2 j+1}
\end{array}
$$

est le carré canonique de $N_{1} \subset N$. Pour montrer que les deux couples sont isomorphes, il suffit de montrer que $N_{1} \subset N$ et $\widetilde{N}_{1} \subset \tilde{N}$ ont même carré canonique [Popa1, 6.6].

Montrons que $(\mathcal{C})$ et $(\widetilde{\mathcal{C}})$ sont isomorphes:

Nous savons déjà que l'isomorphime $\beta$ envoie $N^{\prime} \cap M_{2 j}$ sur $\widetilde{N}^{\prime} \cap \widetilde{M}_{2 j}$ et $N^{\prime} \cap M_{2 j+1}$ sur $\tilde{N}^{\prime} \cap \widetilde{M}_{2 j+1}$ en conservant la trace. 
Comme $M^{\prime} \cap M_{2 j}$ est l'intersection de $\left(N^{\prime} \cap M_{2 j}\right)$ et $\left(M^{\prime} \cap M_{2 j+1}\right)$, il nous suffit de démontrer que

$$
\beta\left(M^{\prime} \cap M_{2 j+1}\right)=\widetilde{M}^{\prime} \cap \widetilde{M}_{2 j+1} .
$$

Or

$$
M^{\prime} \cap M_{2 j+1}=\gamma_{j}\left(N^{\prime} \cap M_{2 j}\right)
$$

donc

$$
\widetilde{M}^{\prime} \cap \widetilde{M}_{2 j+1}=\widetilde{\gamma}_{j}\left(\widetilde{N}^{\prime} \cap \widetilde{M}_{2 j}\right)=\widetilde{\gamma}_{j} \beta\left(N^{\prime} \cap M_{2 j}\right)
$$

et comme $\gamma_{j}=\beta^{-1} \widetilde{\gamma}_{j} \beta$,

$$
\widetilde{M}^{\prime} \cap \widetilde{M}_{2 j+1}=\beta \gamma_{j}\left(N^{\prime} \cap M_{2 j}\right)=\beta\left(M^{\prime} \cap M_{2 j+1}\right) .
$$

Les deux carrés sont isomorphes.

\section{Produit croisé par une algèbre de Kac de dimension finie.}

Dans cette partie, nous donnons une caractérisation de l'inclusion d'un facteur de type $\mathrm{II}_{1}$ dans son produit croisé par une algèbre de Kac de dimension finie:

Théorème 5.0. Soient $M$ un facteur de type $\mathrm{II}_{1}$, tr sa trace normale finie fidèle normalisée et $N$ un sous-facteur d'indice fini dans $M$. Les propositions suivantes sont équivalentes:

(a) $\quad N$ est de profondeur au plus 2 dans $M$ et $N^{\prime} \cap M$ est égal à $\mathbb{C}$.

(b) $M$ est le produit croisé de $N$ par une action extérieure d'une algèbre de Kac de dimension finie $\mathbb{K}$.

(c) $N$ est la sous-algèbre des point fixes de $M$ sous une action extérieure d'une algèbre de Kac de dimension finie $\widehat{\mathbb{K}}$.

L'équivalence entre (b) est (c) est un résultat de M. Enock et J.-M. Schwartz [ES2]; dans [EN], on trouvera la démonstration de (b) $\Rightarrow$ (a) dans le cas le plus général. Nous nous attacherons ici à construire une algèbre de Kac de dimension finie à partir de l'inclusion d'un sous-facteur dans un facteur de type $\mathrm{II}_{1}$, c'est-à-dire à montrer $(\mathrm{a}) \Rightarrow(\mathrm{c})$.

Soient $M$ un facteur de type $\mathrm{II}_{1}$, tr sa trace normale finie fidèle normalisée et $N$ un sous-facteur d'indice fini $n$ et de profondeur 2 dans $M$. Par construction de base, on obtient la tour de facteurs

$$
N \subset M \stackrel{e_{0}}{\complement} M_{1} \stackrel{e_{1}}{\complement} M_{2} \stackrel{e_{2}}{\complement} \ldots M_{p} \stackrel{e_{p}}{\complement} M_{p+1} \ldots,
$$

la suite des projecteurs de V. Jones et les anti-automorphismes $\gamma_{p}$ définie en 2.2.1. On s'intéressera plus particulièrement à la tour dérivée de l'inclusion $M \subset M_{1}$, aussi on notera $B_{p}=M^{\prime} \cap M_{p}$. On supposera, de plus, que $N^{\prime} \cap M=\mathbb{C}$. 
5.1. La tour dérivée de l'inclusion $M \subset M_{1}$. Comme $N^{\prime} \cap M=\mathbb{C}$, $B_{1}=\gamma_{0}\left(N^{\prime} \cap M\right)=\mathbb{C}$.

Comme l'inclusion de $N$ dans $M$ est de profondeur $2, N^{\prime} \cap M_{2}$ est obtenue par construction de base sur l'inclusion $N^{\prime} \cap M \subset N^{\prime} \cap M_{1}$ [GHJ, 2.4.1 ou 4.6.3], donc $N^{\prime} \cap M_{2}$ est un facteur de dimension finie puisque $[M: N]$ est fini, soit $M_{n}(\mathbb{C})$ où $n=[M: N]$ qui est donc entier. Alors $B_{3}=\gamma_{1}\left(N^{\prime} \cap M_{2}\right)$ est aussi isomorphe au facteur $M_{n}(\mathbb{C})$, plus précisément c'est le facteur $\mathcal{B}\left(H_{\varphi}\right)$, si $\left(B_{2}, H_{\varphi}, J_{\varphi}, \varphi\right)$ est la forme standard de $B_{2}$.

Alors $B_{2}$ est une algèbre de dimension finie $n$, soit $B_{2}=\oplus_{i \in I} M_{n_{i}}(\mathbb{C})$ où $I$ est un ensemble fini et $\sum_{i \in I} n_{i}^{2}=n$. On notera $M_{n_{1}}(\mathbb{C})$ le sous-facteur $\mathbb{C} e_{1}$ de $B_{2}$ (donc $n_{1}=1$ ) et $p_{i}$ le projecteur central de $B_{2}$ tel que $B_{2} p_{i} \cong M_{n_{i}}(\mathbb{C})$. La trace de Markov normalisée sur $B_{2}$, notée $\varphi$, est la restriction de la trace de $M_{2}$, sa valeur sur le projecteur minimal du facteur $B_{2} p_{i}$ est $\frac{n_{i}}{n}$.

Nous allons montrer que $\left(B_{2}, \gamma_{2} \gamma_{1}, \gamma_{1}, n \varphi\right)$ est une algèbre de Kac qui agit sur $M$ en laissant fixes les éléments de $N$.

Nous utiliserons seulement deux réflexions de la tour dérivée:

i) $\gamma_{\varphi}$, l'anti-automorphisme de l'algèbre $B_{3}$ défini par

$$
\gamma_{\varphi}(a)=J_{\varphi} a^{*} J_{\varphi} \quad\left(a \in B_{3}\right)
$$

$\gamma_{\varphi}$ envoie $B_{2}$ dans $B_{2}^{\prime}$.

ii) $\gamma_{H_{\varphi}}$ défini de la même façon à partir de $J_{H_{\varphi}}$ l'involution standard de $B_{3}$, c'est un anti-automorphisme de l'algèbre $B_{2}^{\prime} \cap B_{4}$ qui envoie $B_{2}^{\prime} \cap B_{3}$ sur $B_{3}^{\prime} \cap B_{4}$.

Comme nous ne considérons que des constructions de base à un étage, il n'y a aucun problème de compatibilité de répresentations, de plus les formules de la partie 3 sont encore valables; on peut vérifier directement que ces réflexions conservent le trace de Markov de la tour dérivée.

5.2. Bases de Pimsner-Popa et unités matricielles. Pour appliquer les formules de 3 , nous allons choisir des bases de Pimsner-Popa particulières. La proposition suivante motive ce choix.

Proposition 5.2.1. Soit $N$ un sous-facteur d'indice fini $n$ du facteur $M$, tr la trace normale finie fidèle normalisée sur $M$ et $N \subset M \stackrel{e_{0}}{\subset} M_{1}$ la construction de base.

Si $N$ est de profondeur au plus 2 dans $M$ et que $N^{\prime} \cap M$ est égal à $\mathbb{C}$, on pose:

$$
N^{\prime} \cap M_{1}=\oplus_{i \in I} M_{n_{i}}(\mathbb{C}) \quad \text { et } \quad \mathcal{J}=\left\{K=\left(k, k_{1}, k_{2}\right), k \in I, 1 \leq k_{1}, k_{2} \leq n_{k}\right\} .
$$

Soit $\left\{f_{k_{1}, k_{2}}^{k}=f_{K}, K \in \mathcal{J}\right\}$ une famille d'unités matricielles de $N^{\prime} \cap M_{1}$, où $f_{1,1}^{0}=f_{0}=e_{0}$, alors la famille $\left\{\sqrt{\frac{n}{n_{k}}} f_{K}, K \in \mathcal{J}\right\}$ est une base de Pimsner- 
Popa de $M_{1}$ sur $M$ ainsi qu'une base de Pimsner-Popa de $N^{\prime} \cap M_{1}$ sur $\mathbb{C}$; de même pour $\left\{\sqrt{\frac{n}{n_{k}}}\left(f_{K}\right)^{*}, K \in \mathcal{J}\right\}$.

Démonstration. On vérifie facilement les propriétés de 3.1 .5 car, comme $N^{\prime} \cap M=\mathbb{C}$, sur $N^{\prime} \cap M_{1}$, l'espérance conditionelle sur $M$ est la trace.

\section{Définitions.}

1. On choisira pour base de Pimsner-Popa de $M_{1}$ sur $M$ la base de Pimsner-Popa associée à une famille d'unités matricielles de $N^{\prime} \cap M_{1}$ comme dans la proposition 5.2.1 et on la notera pour simplifier $\left\{\lambda_{s}, 1 \leq\right.$ $s \leq n\}$, où $\lambda_{1}=e_{0}$. On ne souviendra que $\sum_{r=1}^{n} \operatorname{tr}\left(\lambda_{r}\right) \lambda_{r}^{*}=1$ et que $\left\{\lambda_{s}^{*}, 1 \leq s \leq n\right\}$ est aussi une base de Pimsner-Popa de $M_{1}$ sur $M$.

On rappelle que $\left\{n^{1 / 2} \lambda_{s} e_{1}, 1 \leq s \leq n\right\}$ est alors une base de $M_{2}$ sur $M_{1}(1.5 .2 \mathrm{~g})$.

2. Si $B_{2}=\oplus_{i \in I} M_{n_{2}}(\mathbb{C})$ et $\mathcal{J}=\left\{K=\left(k, k_{1}, k_{2}\right), k \in I, 1 \leq k_{1}, k_{2} \leq n_{k}\right\}$, on choisit une famille d'unités matricielles de $B_{2},\left\{f_{k_{1}, k_{2}}^{k}=f_{K}, K \in \mathcal{J}\right\}$ où $f_{1,1}^{0}=e_{1}$. La proposition 5.2.1 permet alors d'affirmer que la famille $\left\{\sqrt{\frac{n}{n_{k}}} f_{K}, K \in \mathcal{J}\right\}$ est une base de Pimsner-Popa de $M_{2}$ sur $\mathbb{C}$ ainsi qui une base de Pimsner-Popa de $B_{2}$ sur $\mathbb{C}$; de même pour la famille $\left\{\sqrt{\frac{n}{n_{k}}}\left(f_{K}\right)^{*}, K \in \mathcal{J}\right\}$.

On voudrait voir $\gamma_{2} \gamma_{1}$ comme un co-produit sur $B_{2}$, or $\gamma_{2} \gamma_{1}$ est un isomorphisme de $B_{2}$ sur $M_{2}^{\prime} \cap M_{4}$ qui est contenu dans $B_{2}^{\prime} \cap B_{4}$; il reste à mettre l'algèbre $B_{2}^{\prime} \cap B_{4}$ dans le produit tensoriel $B_{2} \otimes B_{2}$, c'est l'objet de la proposition suivante qui fixe les notations.

\section{Proposition et Définitions 5.2.2.}

a) L'application $\gamma_{\varphi} \gamma_{1}$ est un isomorphisme de $B_{2}$ sur $B_{2}^{\prime} \cap B_{3}$ qui conserve la trace.

Posons $f_{K}^{\prime}=\gamma_{\varphi} \gamma_{1}\left(f_{K}\right)$.

La famille $\left\{f_{K}^{\prime}, K \in \mathcal{J}\right\}$ est une famille d'unités matricielles de $B_{2}^{\prime} \cap B_{3}$. b) L'application $\gamma_{H_{\varphi}}$ est un isomorphisme de $B_{2}$ sur $B_{3}^{\prime} \cap B_{4}$ qui conserve la trace.

Posons $F_{K}=\gamma_{H_{\varphi}} \gamma_{\varphi}\left(f_{K}\right)$.

La famille $\left\{F_{K}, K \in \mathcal{J}\right\}$ est une famille d'unités matricielles de $B_{3}^{\prime} \cap B_{4}$. c) La famille $\left\{f_{H}^{\prime} F_{K}, H \in \mathcal{J}, K \in J\right\}$ est une base de $B_{2}^{\prime} \cap B_{4}$. L'algèbre $B_{2}^{\prime} \cap B_{4}$ est isomorphe à $B_{2} \otimes B_{2}$ par l'isomorphisme $\theta$ :

$$
\theta\left(f_{H}^{\prime} F_{K}\right)=f_{H} \otimes f_{K}
$$

d) L'application $\gamma_{2} \gamma_{1}$ est un isomorphisme de $B_{2}$ sur $M_{2}^{\prime} \cap M_{4}$ qui envoie $B_{2}$ dans $B_{2}^{\prime} \cap B_{4}$ en conservant la trace. 
Posons $g_{K}=\gamma_{2} \gamma_{1}\left(f_{K}\right)$.

Alors $g_{K}=\sum_{P, Q \in \mathcal{J}} x_{P, Q}^{K} f_{P}^{\prime} F_{Q}$ où les $x_{P . Q}^{K}$ sont des nombres complexes. On posera $\Gamma=\theta \gamma_{2} \gamma_{1}$.

La démonstration de cette proposition est laissée au lecteur.

On peut appliquer les résultats du 3 et obtenir les formules suivantes:

\section{Proposition 5.2.3.}

$$
\begin{aligned}
\forall K \in \mathcal{J}, \quad \gamma_{1}\left(f_{K}\right)=n \sum_{s=1}^{n} E_{M_{1}}\left(e_{1} \lambda_{s} f_{K}\right) e_{1} \lambda_{s}^{*} . \\
f_{K}^{\prime}=\sum_{P \in \mathcal{J}} \sqrt{\frac{n}{n_{p}}} f_{P} \gamma_{1}\left(f_{K}\right) e_{2} \sqrt{\frac{n}{n_{p}}} f_{P}^{*} \\
F_{K}=n \sum_{P \in \mathcal{J}} \sqrt{\frac{n}{n_{p}}} f_{P} e_{2} f_{K} e_{3} e_{2} \sqrt{\frac{n}{n_{p}}} f_{P}^{*} \\
g_{K}=n^{2} \sum_{s=1}^{n} \lambda_{s} e_{1} e_{2} f_{K} e_{3} e_{2} e_{1} \lambda_{s}^{*}
\end{aligned}
$$

Démonstration.

a) C'est la formule (3.2.1) pour $\gamma_{1}$ l'anti-automorphisme de $B_{2}$.

b) C'est la formule (3.2.1) pour $\gamma_{\varphi}$ l'anti-automorphisme de $B_{2}$ qui se simplifie:

$$
\begin{aligned}
f_{K}^{\prime} & =n \sum_{P \in \mathcal{J}} E_{B_{2}}\left(e_{2} \sqrt{\frac{n}{n_{p}}} f_{P} \gamma_{1}\left(f_{K}\right)\right) e_{2} \sqrt{\frac{n}{n_{p}}} f_{P}^{*} \\
& =\sum_{P \in \mathcal{J}} \sqrt{\frac{n}{n_{p}}} f_{P} \gamma_{1}\left(f_{K}\right) e_{2} \sqrt{\frac{n}{n_{p}}} f_{P}^{*} .
\end{aligned}
$$

(c) et (d) cf. formule (3.3.3).

5.3. Définition d'un coproduit sur $B_{2}$. Nous allons préciser les composantes de $g_{K}$ sur la base $\left\{f_{H}^{\prime} F_{K}, H \in \mathcal{J}, K \in \mathcal{J}\right\}$, connaisant l'expression de $\Gamma\left(f_{K}\right)$, nous pourrons vérifier que $\Gamma$ est un co-produit co-associatif.

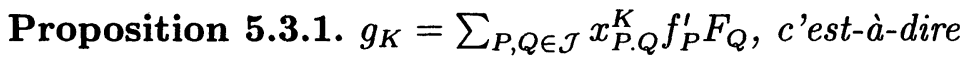

$$
\Gamma\left(f_{K}\right)=\sum_{P, Q \in \mathcal{J}} x_{P, Q}^{K} f_{P} \otimes f_{Q}
$$

où

$$
x_{P, Q}^{K}=\frac{n^{2}}{n_{p} n_{q}} \operatorname{tr}\left(\sum_{r=1}^{n} E_{M_{1}}\left(e_{1} \lambda_{r} f_{P}^{*}\right) f_{K} \lambda_{r}^{*} f_{Q}^{*}\right) .
$$


En particulier $e_{3}=\Gamma\left(e_{1}\right)=\sum_{P \in \mathcal{J}} \frac{1}{n_{p}} \gamma_{1}\left(f_{P}^{*}\right) \otimes f_{P}$.

Démonstration.

Si $P=\left(p ; p_{1}, p_{2}\right)$ et $Q=\left(q ; q_{1}, q_{2}\right)$,

$$
g_{K} f_{P}^{*} F_{Q}^{*}=x_{P, Q}^{K} f_{p_{1} p_{1}}^{\prime p} F_{q_{1} q_{1}}^{q}
$$

et en passant à la trace, on obtient $x_{P, Q}^{K}=\frac{n^{2}}{n_{p} n_{q}} \operatorname{tr}\left(g_{K} f_{P}^{\prime *} F_{Q}^{*}\right)$.

Calculons donc $\operatorname{tr}\left(g_{K} f_{P}^{\prime} F_{Q}\right)$.

$$
\begin{aligned}
f_{P}^{\prime} F_{Q} & =n \sum_{B, C \in \mathcal{J}} \sqrt{\frac{n}{n_{b}}} f_{B} \gamma_{1}\left(f_{P}\right) e_{2}\left(\sqrt{\frac{n}{n_{b}}} f_{B}^{*} \sqrt{\frac{n}{n_{c}}} f_{C}\right) e_{2} f_{Q} e_{3} e_{2} \sqrt{\frac{n}{n_{c}}} f_{C}^{*} \\
& =n \sum_{B \in \mathcal{J}} \sqrt{\frac{n}{n_{b}}} f_{B} \gamma_{1}\left(f_{P}\right) e_{2} f_{Q} e_{3} e_{2} \sqrt{\frac{n}{n_{b}}} f_{B}^{*}
\end{aligned}
$$

$\operatorname{car}\left\{\sqrt{\frac{n}{n_{\mathrm{c}}}} f_{C}, C \in \mathcal{J}\right\}$ est une base de Pimsner-Popa [1.5.2a]. Donc

$$
\begin{aligned}
& \operatorname{tr}\left(g_{K} f_{P}^{\prime} F_{Q}\right)= \\
= & n^{3} \operatorname{tr}\left(\sum_{B \in \mathcal{J}, s=1}^{n} \lambda_{s} e_{1} e_{2} f_{K} e_{3} e_{2}\left(e_{1} \lambda_{s}^{*} \sqrt{\frac{n}{n_{b}}} f_{B} \gamma_{1}\left(f_{P}\right)\right) e_{2} f_{Q} e_{3} e_{2} \sqrt{\frac{n}{n_{b}}} f_{B}^{*}\right) \\
= & n^{3} \operatorname{tr}\left(\sum_{B \in \mathcal{J}, s=1}^{n} \lambda_{s} e_{1} e_{2} f_{K} e_{3} E_{M_{1}}\left(e_{1} \lambda_{s}^{*} \sqrt{\frac{n}{n_{b}}} f_{B} \gamma_{1}\left(f_{P}\right)\right) e_{2} f_{Q} e_{3} e_{2} \sqrt{\frac{n}{n_{b}}} f_{B}^{*}\right) \\
= & n^{2} \operatorname{tr}\left(\sum_{B \in \mathcal{J}, s=1}^{n} \lambda_{s} e_{1} e_{2} f_{K} E_{M_{1}}\left(e_{1} \lambda_{s}^{*} \sqrt{\frac{n}{n_{b}}} f_{B} \gamma_{1}\left(f_{P}\right)\right) f_{Q} e_{3} e_{2} \sqrt{\frac{n}{n_{b}}} f_{B}^{*}\right) .
\end{aligned}
$$

Utilisons la propriété de commutation de la trace et sa propriété de Markov [VJ1, 3.1.7]: on a

$$
\begin{aligned}
& \operatorname{tr}\left(g_{K} f_{P}^{\prime} F_{Q}\right)= \\
& =n \operatorname{tr}\left(\sum_{B \in \mathcal{J}, s=1}^{n} e_{2}\left(\sqrt{\frac{n}{n_{b}}} f_{B}^{*} \lambda_{s} e_{1}\right) e_{2} f_{K} E_{M_{1}}\left(e_{1} \lambda_{s}^{*} \sqrt{\frac{n}{n_{b}}} f_{B} \gamma_{1}\left(f_{P}\right)\right) f_{Q}\right) \\
& =n \operatorname{tr}\left(\sum_{B \in \mathcal{J}, s=1}^{n} E_{M_{1}}\left(\sqrt{\frac{n}{n_{b}}} f_{B}^{*} \lambda_{s} e_{1}\right) e_{2} f_{K} E_{M_{1}}\left(e_{1} \lambda_{s}^{*} \sqrt{\frac{n}{n_{b}}} f_{B} \gamma_{1}\left(f_{P}\right)\right) f_{Q}\right) . \\
& =\operatorname{tr}\left(\sum_{B \in \mathcal{J}, s=1}^{n} E_{M_{1}}\left(\sqrt{\frac{n}{n_{b}}} f_{B}^{*} \lambda_{s} e_{1}\right) f_{K} E_{M_{1}}\left(e_{1} \lambda_{s}^{*} \sqrt{\frac{n}{n_{b}}} f_{B} \gamma_{1}\left(f_{P}\right)\right) f_{Q}\right) .
\end{aligned}
$$


Ecrivons $E_{M_{1}}\left(e_{1} \lambda_{s}^{*} \sqrt{\frac{n}{n_{b}}} f_{B} \gamma_{1}\left(f_{P}\right)\right)$ sur la base $\left\{\lambda_{r}, r=1\right.$ à $\left.n\right\}$ :

$$
\begin{aligned}
& E_{M_{1}}\left(e_{1} \lambda_{s}^{*} \sqrt{\frac{n}{n_{b}}} f_{B} \gamma_{1}\left(f_{P}\right)\right)=\sum_{r=1}^{n} E_{M}\left(E_{M_{1}}\left(e_{1} \lambda_{s}^{*} \sqrt{\frac{n}{n_{b}}} f_{B} \gamma_{1}\left(f_{P}\right)\right) \lambda_{r}\right) \lambda_{r}^{*} \\
& E_{M_{1}}\left(e_{1} \lambda_{s}^{*} \sqrt{\frac{n}{n_{b}}} f_{B} \gamma_{1}\left(f_{P}\right)\right)=\sum_{r=1}^{n} E_{M}\left(e_{1} \lambda_{s}^{*} \sqrt{\frac{n}{n_{b}}} f_{B} \gamma_{1}\left(f_{P}\right) \lambda_{r}\right) \lambda_{r}^{*}
\end{aligned}
$$

Comme $f_{K}$ commute à $M$, on en déduit:

$$
\begin{aligned}
& \operatorname{tr}\left(g_{K} f_{P}^{\prime} F_{Q}\right)= \\
= & \operatorname{tr}\left(\sum_{B \in \mathcal{J}, r, s=1}^{n} E_{M_{1}}\left(\sqrt{\frac{n}{n_{b}}} f_{B}^{*} \lambda_{s} e_{1}\right) E_{M}\left(e_{1} \lambda_{s}^{*} \sqrt{\frac{n}{n_{b}}} f_{B} \gamma_{1}\left(f_{P}\right) \lambda_{r}\right) f_{K} \lambda_{r}^{*} f_{Q}\right) \\
= & \operatorname{tr}\left(\sum_{B \in \mathcal{J}, r, s=1}^{n} E_{M_{1}}\left(\sqrt{\frac{n}{n_{b}}} f_{B}^{*} \lambda_{s} e_{1} E_{M}\left(e_{1} \lambda_{s}^{*} \sqrt{\frac{n}{n_{b}}} f_{B} \gamma_{1}\left(f_{P}\right) \lambda_{r}\right)\right) f_{K} \lambda_{r}^{*} f_{Q}\right) \\
= & \operatorname{tr}\left(\sum_{B \in \mathcal{J}, r, s=1}^{n} E_{M_{1}}\left(\sqrt{\frac{n}{n_{b}}} f_{B}^{*} \lambda_{s} e_{1} E_{M_{1}}\left(e_{1} \lambda_{s}^{*} \sqrt{\frac{n}{n_{b}}} f_{B} \gamma_{1}\left(f_{P}\right) \lambda_{r}\right) e_{1}\right) f_{K} \lambda_{r}^{*} f_{Q}\right)
\end{aligned}
$$

Comme on a:

$$
n \sum_{s+1}^{n} \lambda_{s} e_{1} E_{M_{1}}\left(e_{1} \lambda_{s}^{*} \sqrt{\frac{n}{n_{b}}} f_{B} \gamma_{1}\left(f_{P}\right) \lambda_{r}\right)=\sqrt{\frac{n}{n_{b}}} f_{B} \gamma_{1}\left(f_{P}\right) \lambda_{r}
$$

on en déduit:

$$
\operatorname{tr}\left(g_{K} f_{P}^{\prime} F_{Q}\right)=\frac{1}{n} \operatorname{tr}\left(\sum_{B \in \mathcal{J}, r=1}^{n} E_{M_{1}}\left(\sqrt{\frac{n}{n_{b}}} f_{B}^{*} \sqrt{\frac{n}{n_{b}}} f_{B} \gamma_{1}\left(f_{P}\right) \lambda_{r} e_{1}\right) f_{K} \lambda_{r}^{*} f_{Q}\right) .
$$

Et comme $\sum_{B} \sqrt{\frac{n}{n_{b}}} f_{B}^{*} \sqrt{\frac{n}{n_{b}}} f_{B}=n(1.5 .3 e)$, on peut écrire:

$$
\operatorname{tr}\left(g_{K} f_{P}^{\prime} F_{Q}\right)=\operatorname{tr}\left(\sum_{r=1}^{n} E_{M}\left(\gamma_{1}\left(f_{P}\right) \lambda_{r} e_{1}\right) f_{K} \lambda_{r}^{*} f_{Q}\right) .
$$

Simplifions $E_{M_{1}}\left(\gamma_{1}\left(f_{P}\right) \lambda_{r} e_{1}\right)$ en remplaçant $\gamma_{1}\left(f_{P}\right)$ par

$$
n \sum_{s=1}^{n} E_{M_{1}}\left(e_{1} \lambda_{s} f_{P}\right) e_{1} \lambda_{s}^{*}
$$

On obtient alors

$$
x_{P, Q}^{K}=\frac{n^{2}}{n_{p} n_{q}} \operatorname{tr}\left(g_{K} f_{P}^{\prime *} F_{Q}^{*}\right)=\frac{n^{2}}{n_{p} n_{q}} \operatorname{tr}\left(\sum_{r=1}^{n} E_{M_{1}}\left(e_{1} \lambda_{r} f_{P}^{*}\right) f_{K} \lambda_{r}^{*} f_{Q}^{*}\right) .
$$


On notera $\gamma_{K}\left(f_{P}^{*}\right)=n \sum_{r=1}^{n} E_{M_{1}}\left(e_{1} \lambda_{r} f_{P}^{*}\right) f_{K} \lambda_{r}^{*}$. (Cette notation est cohérente puisque $\left.\gamma_{1}\left(f_{P}\right)=n \sum_{s=1}^{n} E_{M_{1}}\left(e_{1} \lambda_{s} f_{P}\right) e_{1} \lambda_{s}^{*}\right)$. On peut alors écrire

$$
x_{P, Q}^{K}=\frac{n}{n_{p} n_{q}} \operatorname{tr}\left(\gamma_{K}\left(f_{P}^{*}\right) f_{Q}^{*}\right) .
$$

En particulier:

$$
\begin{aligned}
e_{3} & =\gamma_{2} \gamma_{1}\left(e_{1}\right) \\
& =\sum_{P, Q \in \mathcal{J}} \frac{n}{n_{p} n_{q}} \operatorname{tr}\left(f_{Q}^{*} \gamma_{1}\left(f_{P}^{*}\right)\right) f_{P}^{\prime} F_{Q} \\
& =\sum_{P, Q \in \mathcal{J}} \frac{n}{n_{p} n_{q}} \operatorname{tr}\left(f_{Q}^{*} \gamma_{1}\left(f_{P}^{*}\right)\right) f_{P} \otimes f_{Q} .
\end{aligned}
$$

Comme $\left\{\sqrt{\frac{n}{n_{b}}} f_{B}, B \in \mathcal{J}\right\}$ est une base de Pimsner-Popa de $B_{2}$ sur $\mathbb{C}$ et que $\gamma_{1}$ conserve la trace, on peut écrire

$$
e_{3}=\sum_{P \in \mathcal{J}} \frac{1}{n_{p}} f_{P} \otimes \gamma_{1}\left(f_{P}^{*}\right)=\sum_{P \in \mathcal{J}} \frac{1}{n_{p}} \gamma_{1}\left(f_{P}^{*}\right) \otimes f_{P} .
$$

5.4. Dualité entre $A_{1}$ et $B_{2}$. Dans [S], W. Szymanski définit une dualité entre les espaces vectoriels $A_{1}$ et $B_{2}$.

Définition et Proposition 5.4.1. (W. Szymanski) La forme linéaire définie sur $A_{1} \times B_{2}$ par

$$
(a, b)=n^{2} \operatorname{tr}\left(a e_{1} e_{0} b\right) \quad\left(a \in A_{1}, b \in B_{2}\right)
$$

établit une dualité entre $A_{1}$ et $B_{2}$.

Démonstration.

Rappelons d'abord que $A_{1} e_{0}=\mathbb{C}_{0}$ et $e_{1} B_{2}=\mathbb{C} e_{1}$. Alors si, pour $b$ donné dans $B_{2}, n^{2} \operatorname{tr}\left(a e_{1} e_{0} b\right)$ est nul pour tout $a$ de $A_{1}, n^{2} \operatorname{tr}\left(a e_{1} a^{\prime} e_{0} b\right)$ est nul pour tous $a$ et $a^{\prime}$ de $A_{1}$.

Comme $A_{2}=A_{1} e_{1} A_{1}$ et que $e_{0} b$ est un élément de $A_{2}$, nous concluons à la nullité de $\operatorname{tr}\left(e_{0} b b^{*} e_{0}\right)$.

Or comme $E_{M_{1}}\left(b b^{*}\right)$ appartient à l'algèbre $M^{\prime} \cap M_{1}$ qui vaut $\mathbb{C}$, on peut écrire:

$$
0=\operatorname{tr}\left(e_{0} b b^{*} e_{0}\right)=\operatorname{tr}\left(b b^{*} e_{0}\right)=\operatorname{tr}\left(E_{M_{1}}\left(b b^{*}\right) e_{0}\right)=n^{-1} \operatorname{tr}\left(b b^{*}\right) .
$$

La fidélité de la trace permet de conclure à la nullité de $b$.

Nous démontrons de même que, pour $a$ donné dans $A_{1}$, la nullité de $(a, b)$ pour tout $b$ de $B_{2}$ implique la nullité de $a$. 
La proposition suivante nous assure la co-associativité de $\Gamma$.

Proposition 5.4.2. $\Gamma$, le coproduit de $B_{2}$, est le dual du produit de l'algèbre $A_{1}$.

Démonstration.

L'algèbre $A_{1}$ (resp. $B_{2}$ ) est engendrée par $\left\{\lambda_{s}, 1 \leq s \leq n\right\}$ (resp. $\left.\left\{f_{K}, K \in \mathcal{J}\right\}\right)$. Nous allons donc calculer $\left(\lambda_{h} \otimes \lambda_{s},, \Gamma\left(f_{K}\right)\right)$. D'après 5.4 .1 et 5.3 .1 , nous avons

$$
\left(\lambda_{h} \otimes \lambda_{s},, \Gamma\left(f_{K}\right)\right)=n^{4} \sum_{P, Q \in \mathcal{J}} x_{P, Q}^{K} \operatorname{tr}\left(\lambda_{h} e_{1} e_{0} f_{P}\right) \operatorname{tr}\left(\lambda_{s} e_{1} e_{0} f_{Q}\right) .
$$

\section{Lemme 1.}

$$
e_{0} E_{M_{1}}\left(e_{1} \lambda_{r} f_{P}^{*}\right)=n e_{0} E_{M}\left(e_{0} e_{1} \lambda_{r} f_{P}^{*}\right)=n e_{0} \operatorname{tr}\left(e_{0} e_{1} \lambda_{r} f_{P}^{*}\right)
$$

\section{Démonstration.}

La première égalité est une application directe de [PiPo1, lemma 1.2].

Comme $e_{0} e_{1} \lambda_{r} f_{P}^{*}$ appartient à $A_{2}, E_{M}\left(e_{0} e_{1} \lambda_{r} f_{P}^{*}\right)$ appartient à $N^{\prime} \cap M$ donc vaut $\operatorname{tr}\left(e_{0} e_{1} \lambda_{r} f_{P}^{*}\right)$.

Lemme 2. L'élément $\gamma_{K}\left(f_{P}^{*}\right)=n \sum_{r=1}^{n} E_{M_{1}}\left(e_{1} \lambda_{r} f_{P}^{*}\right) f_{K} \lambda_{r}^{*}$ appartient à $B_{2}$.

\section{Démonstration.}

Soit $y$ un élément de $M, \lambda_{r}^{*} y=\sum_{r=1}^{n} E_{M}\left(\lambda_{r}^{*} y \lambda_{s}\right) \lambda_{s}^{*}$, alors comme $f_{K}, f_{P}^{*}$ et $e_{1}$ commutent à $M$, on peut écrire:

$$
\begin{aligned}
\gamma_{K}\left(f_{P}^{*}\right) y & =n \sum_{r=1}^{n} E_{M_{1}}\left(e_{1} \lambda_{r} f_{P}^{*}\right) f_{K} \lambda_{r}^{*} y \\
& =n \sum_{r=1}^{n} E_{M_{1}}\left(e_{1} \lambda_{r} f_{P}^{*}\right) f_{K} E_{M}\left(\lambda_{r}^{*} y \lambda_{s}\right) \lambda_{s}^{*} \\
& =n \sum_{r=1}^{n} E_{M_{1}}\left(e_{1} \lambda_{r} E_{M}\left(\lambda_{r}^{*} y \lambda_{s}\right) f_{P}^{*}\right) f_{K} \lambda_{s}^{*} \\
& =n \sum_{r=1}^{n} E_{M_{1}}\left(e_{1} y \lambda_{s} f_{P}^{*}\right) f_{K} \lambda_{s}^{*} \\
& =y \gamma_{K}\left(f_{P}^{*}\right)
\end{aligned}
$$

donc $\gamma_{K}\left(f_{P}^{*}\right) \in M^{\prime} \cap M_{2}$. 


\section{Lemme 3.}

$$
\sum_{Q \in \mathcal{J}} x_{P, Q}^{K} \operatorname{tr}\left(\lambda_{s} e_{1} e_{0} f_{Q}\right)=\frac{1}{n_{p}} \sum_{r=1}^{n}\left(\lambda_{r}^{*} \lambda_{s}, f_{K}\right) \operatorname{tr}\left(e_{0} e_{1} \lambda_{r} f_{P}^{*}\right) .
$$

\section{Démonstration.}

$$
\sum_{Q \in \mathcal{J}} x_{P, Q}^{K} \operatorname{tr}\left(\lambda_{s} e_{1} e_{0} f_{Q}\right)=\frac{n}{n_{p} n_{q}} \sum_{Q \in \mathcal{J}} \operatorname{tr}\left[\operatorname{tr}\left(\gamma_{K}\left(f_{P}^{*}\right) f_{Q}^{*}\right) f_{Q} \lambda_{s} e_{1} e_{0}\right]
$$

Puisque $\left\{\sqrt{\frac{n}{n_{q}}} f_{Q}^{*}\right\}$ est une base de $B_{2}$ sur $\mathbb{C}$, le Lemme 2 nous permet d'affirmer:

$$
\sum_{Q \in \mathcal{J}} \frac{n}{n_{q}} \operatorname{tr}\left(\gamma_{K}\left(f_{P}^{*}\right) f_{Q}^{*}\right) f_{Q}=\gamma_{K}\left(f_{P}^{*}\right)
$$

On simplifie alors l'expression:

$$
\sum_{Q \in \mathcal{J}} x_{P, Q}^{K} \operatorname{tr}\left(\lambda_{s} e_{1} e_{0} f_{Q}\right)=\frac{1}{n_{p}} \operatorname{tr}\left(e_{0} \gamma_{K}\left(f_{P}^{*}\right) \lambda_{s} e_{1}\right)
$$

En remplaçant $\gamma_{K}\left(f_{P}^{*}\right)$ par son expression, on obtient:

$$
\sum_{Q \in \mathcal{J}} x_{P, Q}^{K} \operatorname{tr}\left(\lambda_{s} e_{1} e_{0} f_{Q}\right)=\frac{n}{n_{p}} \sum_{r=1}^{n} \operatorname{tr}\left(e_{0} E_{M_{1}}\left(e_{1} \lambda_{r} f_{P}^{*}\right) f_{K} \lambda_{r}^{*} \lambda_{s} e_{1}\right) .
$$

Le `emme 1 nous permet d'écrire:

$$
\sum_{Q \in \mathcal{V}} x_{P, Q}^{K} \operatorname{tr}\left(\lambda_{s} e_{1} e_{0} f_{Q}\right)=\frac{n^{2}}{n_{p}} \sum_{r=1}^{n} \operatorname{tr}\left(e_{0} \operatorname{tr}\left(e_{0} e_{1} \lambda_{r} f_{P}^{*}\right) f_{K} \lambda_{r}^{*} \lambda_{s} e_{1}\right)
$$

On en déduit ‘'xilement le résultat annoncé.

Suite de démonistration de la proposition. D'après le Lemme 3, on peut écrire:

$$
\begin{aligned}
\left(\lambda_{h} \otimes \lambda_{s}, \Gamma\left(f_{K}\right)\right) & =\sum_{r=1}^{n}\left(\lambda_{r}^{*} \lambda_{s}, f_{K}\right) \sum_{P \in \mathcal{J}} \frac{n^{4}}{n_{p}} \operatorname{tr}\left(e_{0} e_{1} \lambda_{r} f_{P}^{*}\right) \operatorname{tr}\left(f_{P} \lambda_{h} e_{1} e_{0}\right) \\
& =\sum_{r=1}^{n}\left(\lambda_{r}^{*} \lambda_{s}, f_{K}\right) \sum_{P \in \mathcal{J}} \frac{n^{3}}{n_{p}} \operatorname{tr}\left(n e_{0} \operatorname{tr}\left(e_{0} e_{1} \lambda_{r} f_{P}^{*}\right) f_{P} \lambda_{h} e_{1}\right) \\
& =\sum_{r=1}^{n}\left(\lambda_{r}^{*} \lambda_{s}, f_{K}\right) \sum_{P \in \mathcal{J}} \frac{n^{3}}{n_{p}} \operatorname{tr}\left(e_{0} E_{M_{1}}\left(e_{1} \lambda_{r} f_{P}^{*}\right) f_{P} \lambda_{h} e_{1}\right) .
\end{aligned}
$$


Comme $\left\{\sqrt{\frac{n}{n_{p}}} f_{P}, P \in \mathcal{J}\right\}$ est une base de Pimsner-Popa de $M_{2}$ sur $M_{1}(5.2)$, on peut simplifier l'expression:

$$
\left(\lambda_{h} \otimes \lambda_{s}, \Gamma\left(f_{K}\right)\right)=n^{2} \sum_{r=1}^{n}\left(\lambda_{r}^{*} \lambda_{s}, f_{K}\right) \operatorname{tr}\left(e_{0} e_{1} \lambda_{r} \lambda_{h} e_{1}\right) .
$$

Grâce à $1.5 .2 \mathrm{a}$ et $\mathrm{b}$ et à définition de $\lambda_{s}(5,2)$, on arrive au résultat espéré.

$$
\left(\lambda_{h} \otimes \lambda_{s}, \Gamma\left(f_{K}\right)\right)=n^{2}\left(\lambda_{h} \lambda_{s}, f_{K}\right) \operatorname{tr}\left(e_{0} e_{1}\right)=\left(\lambda_{h} \lambda_{s}, f_{K}\right) .
$$

Corollaire 5.4.3. $\Gamma=\theta \gamma_{2} \gamma_{1}$ est un coproduit co-associatif sur $B_{2}$.

5.5. $\gamma_{1}$ est une co-involution sur $\left(B_{2}, \Gamma\right) \cdot \gamma_{1}$ est, par définition, une involution sur $B_{2}$, pour montrer qu'avec $\Gamma$, elle munit $B_{2}$ d'une structure d'algèbre de Hopf-Von Neumann co-involutive, nous avons besoin d'en savoir plus sur $\gamma_{H_{\varphi}}$.

\section{Lemme 5.5.1.}

a)

$$
\forall P \in \mathcal{J}, \forall Q \in \mathcal{J}, \theta \gamma_{H_{\varphi}} \theta^{-1}\left(f_{P} \otimes f_{Q}\right)=\gamma_{1}\left(f_{Q}\right) \otimes \gamma_{1}\left(f_{P}\right),
$$

c'est-à-dire que modulo l'identification $\theta$ entre $B_{2}^{\prime} \cap B_{4}$ et $B_{2} \otimes B_{2}$,

$$
\gamma_{H_{\varphi}}=\left(\gamma_{1} \otimes \gamma_{1}\right) \sigma
$$

où $\sigma$ est l'automorphisme de $B_{2} \otimes B_{2}$ défini par $\sigma(x \otimes y)=\sigma(y \otimes x)$ [ES1, 1.2.5].

b) $\gamma_{3}(a)=\gamma_{H_{\varphi}}(a) \quad\left(a \in B_{2}^{\prime} \cap B_{4}\right)$.

Démonstration.

a) $\gamma_{H_{\varphi}}\left(f_{P}^{\prime} F_{Q}\right)=$ $=\gamma_{H_{\varphi}}\left(\gamma_{H_{\varphi}} \gamma_{\varphi}\left(f_{Q}\right)\right) \gamma_{H_{\varphi}}\left(\gamma_{\varphi} \gamma_{1}\left(f_{P}\right)\right)=\gamma_{\varphi} \gamma_{1}\left(\gamma_{1}\left(f_{Q}\right)\right) \gamma_{H_{\varphi}} \gamma_{\varphi}\left(\gamma_{1}\left(f_{P}\right)\right)$.

b) D'après la formule 3.2.1, $\left\{\frac{n}{\sqrt{n_{p}}} f_{P} e_{2}, P \in \mathcal{J}\right\}$ étant une base de PimsnerPopa de $M_{3}$ sur $M_{2}$, si $a \in B_{2}^{\prime} \cap B_{4}$,

$$
\gamma_{3}(a)=n \sum_{P \in \mathcal{J}} E_{M_{3}}\left(e_{3} \frac{n}{\sqrt{n_{p}}} f_{P} e_{2} a\right) e_{3} e_{2} \frac{n}{\sqrt{n_{p}}} f_{P}^{*} .
$$

Comme $\left(e_{3} \frac{n}{\sqrt{n_{p}}} f_{P} e_{2} a\right)$ appartient à $B_{4}, E_{M_{3}}\left(e_{3} \frac{n}{\sqrt{n_{p}}} f_{P} e_{2} a\right)$ commute à $M$ donc vaut $E_{B_{3}}\left(e_{3} \frac{n}{\sqrt{n_{p}}} f_{P} e_{2} a\right)$, on a alors

$$
\gamma_{3}(a)=n \sum_{P \in \mathcal{J}} E_{B_{3}}\left(e_{3} \frac{n}{\sqrt{n_{p}}} f_{P} e_{2} a\right) e_{3} e_{2} \frac{n}{\sqrt{n_{p}}} f_{P}^{*} .
$$


C'est la formule 3.2.1 pour $\gamma_{H_{\varphi}}$ défini sur $B_{2}^{\prime} \cap B_{4}$, ainsi $\gamma_{3}$ et $\gamma_{H_{\varphi}}$ coïncident $\operatorname{sur} B_{2}^{\prime} \cap B_{4}$.

Proposition 5.5.2. Le triplet $\left(B_{2}, \Gamma, \gamma_{1}\right)$ est une algèbre de Hopf-Von Neumann co-involutive.

\section{Démonstration.}

D'après 5.5.1a, $\left(\gamma_{1} \otimes \gamma_{1}\right) \sigma \Gamma \gamma_{1}=\left.\theta \gamma_{H_{\varphi}} \gamma_{2}\right|_{B_{2}}$. Comme $\gamma_{2}\left(B_{2}\right)$ est contenu dans $B_{2}^{\prime} \cap B_{4}$, d'après $5.5 .1 \mathrm{~b},\left.\gamma_{H_{\varphi}} \gamma_{2}\right|_{B_{2}}=\left.\gamma_{3} \gamma_{2}\right|_{B_{2}}$. La propriété (2.2.1a) des anti-automorphismes nous permet alors d'écrire:

$$
\left(\gamma_{1} \otimes \gamma_{1}\right) \sigma \Gamma \gamma_{1}=\theta \gamma_{2} \gamma_{1}=\Gamma \text {. }
$$

Le triplet $\left(B_{2}, \Gamma, \gamma_{1}\right)$ est une algèbre de Hopf-Von Neumann co-involutive [ES1, 1.2.5].

Remarque. On peut vérifier facilement que $\gamma_{1}$ est la co-involution définie par la dualité entre $A_{1}$ et $B_{2}$.

Proposition 5.5.3. $\gamma_{1}$, la co-involution sur $B_{2}$, est le dual de l'involution de $A_{1}$.

Démonstration.

Pour $\lambda_{r}$ dans $A_{1}$ et $f_{K}$ dans $B_{2}$, on a

$$
\begin{aligned}
\left(\lambda_{r}, \gamma_{1}\left(f_{K}\right)\right) & =n^{3} \sum_{s=1}^{n} \operatorname{tr}\left(\lambda_{r} e_{1} e_{0} E_{M_{1}}\left(e_{1} \lambda_{s} f_{K}\right) e_{1} \lambda_{s}^{*}\right) \\
& =n^{3} \sum_{s=1}^{n} \operatorname{tr}\left(\lambda_{r} E_{M}\left(e_{0} E_{M_{1}}\left(e_{1} \lambda_{s} f_{K}\right)\right) e_{1} \lambda_{s}^{*}\right) .
\end{aligned}
$$

Comme $e_{0} E_{M_{1}}\left(e_{1} \lambda_{s} f_{K}\right)$ appartient à $N^{\prime} \cap M_{1}$, on a

$$
\begin{aligned}
\left(\lambda_{r}, \gamma_{1}(b)\right) & =n^{3} \sum_{s=1}^{n} \operatorname{tr}\left(\lambda_{r} \operatorname{tr}\left(e_{0} e_{1} \lambda_{s} f_{K}\right) e_{1} \lambda_{s}^{*}\right) \\
& =n^{3} \sum_{s=1}^{n} \operatorname{tr}\left(\lambda_{r} e_{1} \operatorname{tr}\left(f_{K} e_{0} e_{1} \lambda_{s}\right) \lambda_{s}^{*}\right)
\end{aligned}
$$

Comme $\left\{\lambda_{s}, 1 \leq s \leq n\right\}$ est une base de Pimsner-Popa de $A_{1}$ sur $\mathbb{C}$, on peut écrire:

$$
\left(\lambda_{r}, \gamma_{1}(b)\right)=n^{3} \operatorname{tr}\left(\lambda_{r} e_{1} E_{M_{1}}\left(f_{K} e_{0} e_{1}\right)\right)=n^{2} \operatorname{tr}\left(\lambda_{r} E_{M_{1}}\left(f_{K} e_{0} e_{1}\right)\right) .
$$

On en déduit que

$$
\left(\lambda_{r}, \gamma_{1}(b)\right)=n^{2} \operatorname{tr}\left(f_{K} e_{0} e_{1} \lambda_{r}\right)=\overline{\left(\lambda_{r}^{*}, f_{K}^{*}\right)} .
$$


5.6. $\left(B_{2}, \Gamma, \gamma_{1}, n \varphi\right)$ est une algèbre de Kac. Nous allons montrer que $n \varphi$ est un poids de Haar sur $\left(B_{2}, \Gamma, \gamma_{1}\right)$ en utilisant le théorème 6.3.5 de [ES2].

Lemme 5.6.1. $\forall K \in \mathcal{J}, g_{K} e_{1}=e_{1} F_{K}$, c'est-à-dire

$$
\forall a \in B_{2}, \quad \Gamma(a)\left(e_{1} \otimes 1\right)=e_{1} \otimes a .
$$

Démonstration.

Nous utilisons la formule de la Proposition 5.3.1 et la notation déjà utilisée $\gamma_{K}\left(f_{P}^{*}\right)=n \sum_{r=1}^{n} E_{M_{1}}\left(e_{1} \lambda_{r} f_{P}^{*}\right) f_{K} \lambda_{r}^{*}$, en particulier

$$
\gamma_{K}\left(e_{1}\right)=\sum_{r=1}^{n} \operatorname{tr}\left(\lambda_{r}\right) f_{K} \lambda_{r}^{*}=f_{K}
$$

Comme $g_{K}=\sum_{P \in \mathcal{J}} \frac{1}{n_{p}} f_{P} \otimes \gamma_{K}\left(f_{P}^{*}\right), g_{K} e_{1}=e_{1} \otimes \gamma_{K}\left(e_{1}\right)=e_{1} \otimes f_{K}$.

Lemme 5.6.2. $\forall K \in \mathcal{J}, \theta\left(g_{K}\right)\left(1 \otimes e_{1}\right)=f_{K} \otimes e_{1}$, c'est-à-dire

$$
\forall a \in B_{2}, \quad \Gamma(a)\left(1 \otimes e_{1}\right)=a \otimes e_{1} .
$$

Démonstration.

Comme $\theta\left(g_{K}\right)=\sum_{P, Q \in \mathcal{J}} \frac{n^{2}}{n_{p} n_{q}} \operatorname{tr}\left(\sum_{r=1}^{n} E_{M_{1}}\left(e_{1} \lambda_{r} f_{P}^{*}\right) f_{K} \lambda_{r}^{*} f_{Q}^{*}\right) f_{P} \otimes f_{Q}$, on a:

$$
\theta\left(g_{K}\right)\left(1 \otimes e_{1}\right)=\sum_{P \in \mathcal{J}} \frac{n}{n_{p}} \operatorname{tr}\left(n \sum_{r=1}^{n} E_{M_{1}}\left(f_{K} \lambda_{r}^{*} e_{1}\right) e_{1} \lambda_{r} f_{P}^{*}\right) f_{P} \otimes e_{1} .
$$

Or, puisque $\left\{n^{1 / 2} \lambda_{r} e_{1}, 1 \leq r \leq n\right\}$ est une base de Pimsner-Popa de $M_{2}$ sur $M_{1}$,

$$
n \sum_{r=1}^{n} E_{M_{1}}\left(f_{K} \lambda_{r}^{*} e_{1}\right) e_{1} \lambda_{r}=n \sum_{r=1}^{n} E_{M_{1}}\left(f_{K} \lambda_{r} e_{1}\right) e_{1} \lambda_{r}^{*}=f_{K}
$$

Alors,

$$
\sum_{P \in \mathcal{J}} \frac{n}{n_{p}} \operatorname{tr}\left(n \sum_{r=1}^{n} E_{M_{1}}\left(f_{K} \lambda_{r}^{*} e_{1}\right) e_{1} \lambda_{r} f_{P}^{*}\right) f_{P}=\sum_{P \in \mathcal{J}} \frac{n}{n_{p}} \operatorname{tr}\left(f_{K} f_{P}^{*}\right) f_{P}=f_{K}
$$

et l'égalité est démontrée.

Proposition 5.6.3. $\left(B_{2}, \Gamma, \gamma_{1}, n \varphi\right)$ est une algèbre de Kac de dimension finie. 
Démonstration.

On a vu dans la Proposition 5.3.1 que le projecteur central de $B_{2}, e_{1}$, vérifie:

$$
\Gamma\left(e_{1}\right)=\sum_{P=\left(p ; p_{1}, p_{2}\right) \in \mathcal{J}} \frac{1}{n_{p}} \gamma_{1}\left(f_{p_{2}, p_{1}}^{p}\right) \otimes f_{p_{1}, p_{2}}^{p} .
$$

Le Théorème 6.3.5. de [ES2] et les Lemmes 5.6.1 et 2 permettent de conclure. Remarque. L'algèbre $A_{1}$ munie du co-produit dual du produit de l'algèbre $B_{2}$, de la co-involution duale de l'involution de $B_{2}$ et du poids $n$ tr est l'algèbre de Kac duale de l'algèbre $\left(B_{2}, \Gamma, \gamma_{1}, n \varphi\right)$ [ES1, 6.9.9].

5.7. Une action de $\left(B_{2}, \Gamma, \gamma_{1}, n \varphi\right)$ sur $M$. Il nous reste à faire agir l'algèbre de Kac sur $M$.

\section{Proposition 5.7.1.}

a) Si $N_{1}$ est la première algèbre d'un tunnel construit dans $N \subset M$, c'est$\grave{a}$-dire que $N_{1} \subset N \subset M$ est la construction de base, soit $\nu=\gamma_{0} \gamma_{1}$ l'isomorphisme de $B_{2}$ sur $N_{1}^{\prime} \cap M,\left\{\nu\left(f_{K}\right), K \in \mathcal{J}\right\}$ est une famille d'unités matricielles de $N_{1}^{\prime} \cap M$ et, à une constante multiplicative près, une base de Pimsner-Popa de $M$ sur $N$.

b) Soit $\beta$ le morphisme de $M$ dans $M \otimes B_{2}$ défini par:

$$
\text { si } y \in N \text { et } K \in \mathcal{J}, \quad \beta\left(y \nu\left(f_{K}\right)\right)=(y \otimes 1)(\nu \otimes i) \Gamma\left(f_{K}\right)
$$

$\beta$ est une action de $B_{2}$ sur $M$ dont l'algèbre des point fixes est $N$.

c) $\nu$ se prolonge en un morphisme normal de $B_{2}$ dans $M$ qui vérifie:

$$
\nu(1)=1 \text { et } \beta \nu=(\nu \otimes i) \Gamma .
$$

Démonstration.

a) C'est la Proposition 5.2.1.

b) $\beta$ est une action car c'est un morphisme injective de $M$ dans $M \otimes B_{2}$ qui vérifie

$$
\beta(1)=1 \quad \text { et } \quad(\beta \otimes i) \beta=(i \otimes \Gamma) \beta . \quad[\mathbf{E} 1,1.1]
$$

En effet si $y \in N$,

$$
\begin{aligned}
(i \otimes \Gamma) \beta\left(y \nu\left(f_{K}\right)\right) & =(y \otimes 1 \otimes 1)(i \otimes \Gamma)(\nu \otimes i) \Gamma\left(f_{K}\right) \\
& =(y \otimes 1 \otimes 1)(\nu \otimes i \otimes i)(i \otimes \Gamma) \Gamma\left(f_{K}\right) \\
& =(y \otimes 1 \otimes 1)(\nu \otimes i \otimes i)(\Gamma \otimes i) \Gamma\left(f_{K}\right)
\end{aligned}
$$




$$
\begin{aligned}
& =\sum_{P, Q \in \mathcal{J}} x_{P, Q}^{K}\left[(y \otimes 1)(\nu \otimes i) \Gamma\left(f_{P}\right)\right] \otimes f_{Q} \\
& =(\beta \otimes i)\left[(y \otimes 1)(\nu \otimes i) \Gamma\left(f_{K}\right)\right] \\
& =(\beta \otimes i) \beta\left(y \nu\left(f_{K}\right)\right) .
\end{aligned}
$$

Comme $1=\sum_{K \in \mathcal{J}} f_{K}, \beta$ laisse fixe les éléments de $N$. D'autre part, comme $[M: N]=n=\left[M: N^{\beta}\right]$, l'inclusion $N^{\beta} \subset N$ implique l'égalité.

c) résulte de la définition de $\nu$ et $\beta$.

La Proposition 5.7.1 et le Théorème 5.2 de [ES2] permettent de conclure:

Théorème 5.7.2. Soient $M$ un facteur de type $\mathrm{II}_{1}$, tr sa trace normale finie fidèle normalisée et $N$ un sous-facteur d'indice fini dans $M$. Si $N$ est de profondeur au plus 2 dans $M$ et $N^{\prime} \cap M$ est égal à $\mathbb{C}, N$ est la sousalgèbre des points fixes de $M$ sous l'action extérieure $\beta$ de l'algèbre de Kac de dimension finie $\left(M^{\prime} \cap M_{2}, \theta \gamma_{2} \gamma_{1}, \gamma_{1}, n \varphi\right)$.

L'action $\beta$ est extérieure puisque $N^{\prime} \cap M=\mathbb{C}$.

5.8. Remarque: cas d'un groupe fini. Le cas où $N^{\prime} \cap M_{1}$ est abélien peut se traiter directement, en effet le groupe $G$ apparaît comme le quotient du normalisateur de $N$ par le groupe unitaire de $N$. Un résultat de Sutherland repris dans la thèse de $\mathrm{V}$. Jones permet de conclure [thèse $\mathrm{VJ}, 4.1 .7$ ].

\section{References}

[E1] M. Emock, Produit croisé d'une algèbre de von Neumann par une algèbre de Kac, Journal of Functional Analysis, 26(1) (1977).

[EN] M. Enock et R. Nest, en préparation.

[ES1] M. Enock et Jean-Marie Schwartz, Kac algebras and duality of locally compact groups, à paraître chez Springer.

[ES2] M. Enock et Jean-Marie Schwartz, Produit croisé d'une algèbre de von Neumann par une algèbre de Kac, II, P.R.I.M.S.K.U., 16(1) (1980).

[GHJ] F.M. Goodman, P. de la Harpe et V.F.R. Jones, Coxeter Graphs and Towers of algebras, MSRI Publications, 14.

[I1] N. Izumi, Application of Fusion Rules to Classification of subfactors, Publ. RIMS, Kyoto Univ., 27 (1991), 953-994.

[I2] N. Izumi, On subalgebras of non AF-algebras with finite Wakatani index. I. Cuntz algebras, preprint.

[L1] R. Longo, Index of subfactors and statistics of quantum fields I, Comm. Math. Phys., 126 (1989), 217-247.

[L2] R. Longo, A duality for Hopf algebras and subfactors I, preprint 1992.

[O] Quantum Symmetry, differential geometry of finite graphs and classification of subfactors, Lectures given at University of Tokyo by Adrian Ocneanu, notes recorded by Yasuyuki Kawahigashi.

PiPo1] M. Pimsner et S. Popa, Entropy and index for subfactors, Ann. Scient. ENS, 19 (1986), 57-106. 
PiPo2] M. Pimsner et S. Popa, Iterating the basic construction, Trans. A.M.S., 310(1) (1988), 127-134.

Popa1] S. Popa, Classification of subfactors: reduction to commuting squares, Invent. Math., 101 (1990), 19-43.

Popa2] S. Popa, Sur la classification des sous-facteurs d'indice fini du facteur hyperfin.

[S] W. Szymanski, Finite index subfactors and Hopf algebra crossed products, à paraître dans "Proceedings of the AMS".

[VJ1] V. Jones, Index for subfactors, Invent. Math., 72 (1983), 1-25.

sse VJ] V. Jones, Actions of finite groups on the hyperfinite type $\mathrm{II}_{1}$ factor, Memoirs of AMS, 237.

Received February 15, 1993 and revised March 5, 1993.

UNIVERSITÉ PARIS-SUD

91405 Orsay, France

E-mail address: Marie-Claude.David@math.u-psud.fr 

Rosa M. Miró-Roig, Singular moduli spaces of stable vector bundles on $\mathbf{P}^{3}$

Hitoshi Moriyoshi and Toshikazu Natsume, The Godbillon-Vey Cyclic Cocycle and Longitudinal Dirac Operators

J.C. Naranjo, The positive dimensional fibres of the Prym map

Artur Nicolau and Arne Stray, Nevanlinna's coefficients and Douglas al-

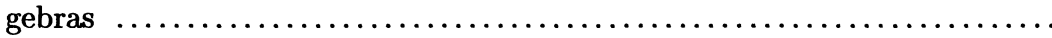

K.K. Park, Entropy of a skew product with a $Z^{2}$-action $\ldots \ldots \ldots \ldots \ldots .227$

María Cristina Pereyra, Sobolev spaces on Lipschitz curves ......... 553

T. Sano, Commuting co-commuting squares and finite dimensional Kac algebras

H.B. Thompson, Second order ordinary differential equations with fully nonlinear two point boundary conditions

H.B. Thompson, Second order ordinary differential equations with fully nonlinear two point boundary conditions II

F. $\mathrm{Xu}$, The flat part of non-flat orbifolds

Hidenobu Yoshida, A type of uniqueness for the Dirichlet problem on a half-space with continuous data 


\section{PACIFIC JOURNAL OF MATHEMATICS}

Volume $172 \quad$ No. $2 \quad$ February 1996

On the failure cycles for the quadratic normality of a projective variety

307

EdoARdo BaLlico

On the minimal free resolution of general embeddings of curves

315

EdOARDO BALlico

On normality of the closure of a generic torus orbit in $G / P$

321

ROMUALD DABROWSKI

Paragroupe d'Adrian Ocneanu et algèbre de Kac

331

MARIE-Claude DAVID

Irreducibility and dimension theorems for families of height 3 Gorenstein algebras

SuSAN J. DiESEL

On the cohomology of the Lie algebra $L_{2}$

ALICE FIALOWSKI

Generic differentiability of convex functions on the dual of a Banach space

John R. Giles, P. S. Kenderov, WarRen Brian Moors and S. D.

SCIFFER

Moon hypersurfaces and some related existence results of capillary hypersurfaces

without gravity and of rotational symmetry

FEI-TSEN LIANG

Stable relations. II. Corona semiprojectivity and dimension-drop $C^{*}$-algebras

TERRY ATHERTON LORING

Singular moduli spaces of stable vector bundles on $\mathbf{P}^{3}$

Rosa M. MIRó-ROIG

The Godbillon-Vey cyclic cocycle and longitudinal Dirac operators

Hitoshi MoRIYOSHI and TOSHIKAZU NATSUME

Nevanlinna's coefficients and Douglas algebras

ARTUR NiCOLAU and ARNE STRAY

Sobolev spaces on Lipschitz curves

MARÍA CRISTINA PEREYRA

A type of uniqueness for the Dirichlet problem on a half-space with continuous data 